\title{
Neuroinflammation creates an immune regulatory niche at the meningeal lymphatic vasculature near the cribriform plate.
}

\section{Zsuzsanna Fabry ( $\sim$ zfabry@wisc.edu )}

University of Wisconsin-Madison

Martin Hsu

University of Wisconsin Madison

Collin Laaker

University of Wisconsin Madison

Andy Madrid

University of Wisconsin Madison

Melinda Herbath

University of Wisconsin Madison

Yun Hwa Choi

University of Wisconsin Madison

Matyas Sandor

University of Wisconsin Madison

\section{Article}

Keywords: meningeal lymphatic vessels, neuroinflammation, neuroinflammatory diseases

Posted Date: June 1st, 2021

DOl: https://doi.org/10.21203/rs.3.rs-543816/v1

License: (c) (i) This work is licensed under a Creative Commons Attribution 4.0 International License.

Read Full License

Version of Record: A version of this preprint was published at Nature Immunology on March 28th, 2022. See the published version at https://doi.org/10.1038/s41590-022-01158-6. 
Neuroinflammation creates an immune regulatory niche at the meningeal lymphatic vasculature near the cribriform plate.

Martin Hsu ${ }^{1}$, Collin Laaker ${ }^{1}$, Andy Madrid ${ }^{1}$, Melinda Herbath ${ }^{2}$, Yun Hwa Choi ${ }^{3}$, Matyas Sandor ${ }^{2,4}$, \& Zsuzsanna Fabry ${ }^{2,4^{*}}$

${ }^{1}$ Neuroscience Training Program, University of Wisconsin Madison, Madison WI 53705, USA

${ }^{2}$ Department of Pathology and Laboratory Medicine, School of Medicine and Public Health, University of Wisconsin Madison, Madison WI 53705, USA

${ }^{3}$ School of Pharmacy, University of Wisconsin Madison, Madison WI 53705, USA

${ }^{4}$ Co-senior authors

*Corresponding Author: zfabry@wisc.edu 


\begin{abstract}
Meningeal lymphatic vessels residing in the dural layer above the sinuses of the brain, meninges at the base of the brain, and near the cribriform plate have all been shown to drain fluid, cells, and antigens. We have previously reported that meningeal lymphatics near the cribriform plate undergo VEGFR3-dependent lymphangiogenesis during experimental autoimmune encephalomyelitis (EAE) to facilitate excess drainage. Using single-cell RNA sequencing (scRNA-seq), we report that neuroinflammation changes the phenotype and function of cribriform plate lymphatic endothelial cells (cpLECs). Upregulation of genes involved in antigen presentation, adhesion to leukocytes, and immunoregulatory molecules were verified by flow cytometry and functional assays. The inflamed cpLECs retain dendritic cells and to lesser extent CD4 T cells, creating an immune-regulatory niche that represents a previously underappreciated interface in the regulation of neuroinflammation. Additionally, the discontinuity of the arachnoid membrane near cpLECs provides unrestricted access to the cerebrospinal fluid (CSF) for immune surveillance. These findings may lead to new therapeutic approaches to neuroinflammatory diseases.
\end{abstract}




\section{Introduction}

The network of lymphatic vessels residing in the meninges surrounding the central nervous system (CNS) have been characterized, and drainage of fluid and macromolecules through these lymphatic vessels has been studied during steady-state conditions ${ }^{1-4 ;}{ }^{5}$. Because meningeal lymphatics are hypothesized to facilitate drainage of CSF, they have also been shown to play significant roles in pathology where drainage of excess fluid, cells, and/or antigens are critical, including experimental autoimmune encephalomyelitis (EAE) ${ }^{4,6}$, glioblastoma ${ }^{7-9}$, Alzheimer's disease ${ }^{10}$, aging ${ }^{10,11}$, Parkinson's disease ${ }^{12}$, stroke ${ }^{13}$, cerebrovascular disease ${ }^{14}$, and several other models ${ }^{15}$. Global inhibition of meningeal lymphatic function using the VEGFR3 tyrosine kinase inhibitor MAZ51 or locally using photoablation prior to EAE onset can reduce CNS-derived autoantigen drainage and delay EAE onset ${ }^{4,6}$, worsens pathology in a mouse model of Alzheimer's disease ${ }^{10}$, and in traumatic brain injury ${ }^{16}$. Similarly, augmenting meningeal lymphatic function through VEGFC can improve anti-tumor immunity ${ }^{7,8}$ and improve cognitive function during aging ${ }^{10}$. These data suggest that manipulation of lymphatic drainage can influence disease outcome depending on the context, highlighting the significance of meningeal lymphatic vessels in the drainage of fluid, antigens, and cells of the CNS.

In addition to the conventional roles of lymphatics in drainage, unbiased approaches such as single-cell RNA sequencing have highlighted novel functions of lymphatic endothelial cells (LECs) in the lymph nodes to directly engage in leukocyte crosstalk and regulation. LECs of the lymph nodes are heterogeneous, containing both afferent and efferent lymphatics that are structurally organized and consequently specialized for different functions such as cell-cell interactions with other resident lymph node cells, antigen processing and presentation with leukocytes from the lymph, pathogen interactions, and remodeling ${ }^{17,18}$. Functionally, lymph node-resident LECs directly regulate leukocytes through cell-cell signaling and tolerance induction through PD-L1/PD-1, as well as acting as non-professional antigen presenting cells through antigen processing, archival, and presentation ${ }^{19-25}$. These data suggest that LECs play a much more significant and direct role in regulating immunity through leukocyte binding and crosstalk than previously hypothesized, and have important implications for targeting lymphatic function during autoimmunity and/or antitumor immunity ${ }^{19}$. While these functions have been specifically attributed to lymph node-resident LECs, it is not known whether LECs upstream that directly drain the brain parenchyma during neuroinflammation could display these functions.

We tested if lymphatics near the cribriform plate can adapt to a neuroinflammatory microenvironment, display immune regulatory functions, and engage in leukocyte crosstalk and if so, how this may influence immunity. We focussed on lymphatics near the cribriform plate due to their unique ability to undergo lymphangiogenesis during experimental 
autoimmune encephalomyelitis (EAE) ${ }^{4}$, a mouse model of multiple sclerosis (MS). Additionally, they have unique access to pro-lymphangiogenic factors from the CSF compartment, suggesting that they may be in a prime anatomical position to sample CSF and engage in leukocyte crosstalk. To characterize cribriform plate lymphatic endothelial cells (cpLECs), we FACS sorted for cpLECs to perform single-cell RNA sequencing (scRNA-seq) and compared naive cpLECs with cpLECs during EAE. We found that during EAE, cpLECs upregulate genes involved in leukocyte crosstalk including genes of canonical lymphatic function such as leukocyte adhesion/chemotaxis, as well as novel functions such as antigen processing and presentation, and regulation of leukocyte activation. Inflamed cpLECs increase their ability to bind and retain $\mathrm{CD} 11 \mathrm{c}^{\text {high }} \mathrm{CD} 11 \mathrm{~b}^{+}$dendritic cells and $\mathrm{CD}^{+} \mathrm{T}$ cells, contain intracellular CNS-derived antigens, express $\mathrm{MHC}$ II, and increase the expression of immunoregulatory proteins such as PD-L1 in an IFN-Y-dependent fashion. Additionally, cpLECs have unique access to the CSF compartment due to gaps in the arachnoid barrier separating the cpLECs from the subarachnoid space, increased fluid accumulation near the cribriform plate relative to other regions, and their expression of the water channel AQP-1. These data implicate lymphangiogenic cpLECs as a potential site of immune regulation through leukocyte binding/crosstalk and correlate with their prime anatomical location to sample the CSF compartment. Furthermore, these data reveal for the first time that similar to lymph node-resident LECs that can engage in direct leukocyte crosstalk and regulation during steady-state, cpLECs can acquire these phenotypes during EAE in response to cues from the inflammatory CNS microenvironment. Altogether, this suggests that meningeal lymphatics near the cribriform plate that directly sample CSF play a regulatory role in immunity during neuroinflammation. 


\section{Results}

\section{CpLECs display a unique phenotypic signature during neuroinflammation.}

We have previously demonstrated that neuroinflammation drives extensive VEGFR3-dependent lymphangiogenesis near the cribriform plate, which can functionally contribute to the drainage of CNS-derived antigens, cells, and fluid ${ }^{4}$. Because cpLECs can undergo remodeling and expansion in response to the changing microenvironment induced by neuroinflammation, we first asked what other phenotypic changes cpLECs may undergo during EAE. To characterize the differences between cpLECs in steady-state and in neuroinflammatory conditions, we first looked to measure and phenotype cpLECs by flow cytometry between healthy and EAE mice (Supplementary Figure 1A-C) after generating a single cell suspension of the cribriform plate region (Figure 1A). CpLECs were identified as Podoplanin $^{+}$and $\mathrm{CD} 31^{+}$after gating for live Ghost ${ }^{\text {negative }}$ singlets and excluding CD $45^{\text {intermediate }}$ microglia and CD45 ${ }^{\text {high }}$ leukocytes as previously described ${ }^{2,8,10,26}$ (Supplementary Figure 1A - B). Quantitation of the average number of cpLECs reveals an approximately 5-fold increase in cell number during EAE compared to healthy, validating EAE-induced LEC cell expansion (Supplementary Figure 1C). These data confirm that cpLECs undergo expansion with increased cell numbers and are not merely due to widening of vessels ${ }^{4}$. Further characterization of the excluded $\mathrm{CD} 45^{\text {intermediate }}$ or CD45 ${ }^{\text {high }}$ cells confirms that we have excluded CD45 $5^{\text {intermediate }} \mathrm{CD} 11 \mathrm{~b}^{+}$microglia and CD45 $5^{\text {high }}$ leukocytes including macrophages, dendritic cells, T cells, and B cells (Supplementary Figure 2). Furthermore, gating on CD31 ${ }^{+}$ endothelial cells confirms that lymphatic endothelial cells in this gate express both Podoplanin and Lyve-1.

After validating the isolation of cpLECs for flow cytometry (Figure 1A, Supplementary Figure 2) and confirming our initial results of lymphangiogenesis (Supplementary Figure 1), we next asked if cpLECs undergo any other phenotypic changes in response to neuroinflammation. We processed cpLECs for single-cell RNA sequencing (scRNA-seq) after cell sorting (Figure 1A-J) in which cpLECs were sorted using the LEC markers CD31 and Podoplanin after gating for live Ghost ${ }^{\text {negative }}$ singlets and excluding CD45 $5^{\text {high }}$ leukocytes as previously described ${ }^{2,8,10,26}$. After sorting for cpLECs and pooling cells from 5 naïve C57BL/6J control mice separately from $5 \mathrm{EAE} \mathrm{C57BL/6J}$ mice, the cpLECs were processed with 10x Genomics Chromium Single Cell Gene Expression Assay and sequenced using the Novaseq 6000 (Figure 1A). Non-LECs that appeared in our analysis were excluded, which consisted primarily of autofluorescent cells in the olfactory epithelium ${ }^{27}$ that lacked RNA expression of Prox-1 and CD31 by scRNA-seq. The t-SNE plot of healthy vs. EAE cells revealed that these two populations of cpLECs separate almost exclusively into different clusters, suggesting unique phenotypes between healthy and EAE cells (Figure 1B). Traditional K-means 
clustering grouped both healthy and EAE cpLECs into three clusters (Figure 1C), with cluster 1 consisting primarily of healthy cells (99.84\%) (Figure 1D), and clusters 2 (Figure 1E) and 3 (Figure 1F) consisting primarily of EAE cells ( $97.87 \%$ for cluster $2,66.27 \%$ for cluster 3$)$. scRNA-seq revealed that cluster 1 contained 341 differentially expressed upregulated genes (* FDR $<0.1$, Methods) and 406 differentially expressed down-regulated genes; cluster 2 contained 286 differentially expressed upregulated and 393 differentially expressed down-regulated genes, and cluster 3 contained 90 differentially expressed upregulated genes with no differentially expressed down-regulated genes when locally distinguishing between the other clusters. Representative volcano plots for each of the three clusters highlight the top 50 most upregulated and downregulated genes (Supplementary Figure 3A, C, E). Gene enrichment analysis of cluster 1 revealed gene pathways associated with baseline lymphatic endothelial cell functions, such as catabolic processes, regulation of vasculature development, and cell-cell adhesion, consistent with its makeup of primarily naive cells (Figure 1D). Cluster 2, consisting primarily of EAE cpLECs, were enriched in gene pathways associated with a more activated state (Figure 1E) with genes involved in leukocyte crosstalk. The majority of cluster 3 consisted of EAE cpLECs (66.27\%) enriched in proliferation genes, consistent with our previous data of lymphangiogenesis by $\operatorname{cpLECs}^{4}$ (Figure 1F). Additionally, these data suggest that steady-state cpLECs undergo a baseline level of proliferation to maintain lymphatic cell numbers, and neuroinflammation drives lymphangiogenesis by increasing cell proliferation.

ScRNA-seq validated our initial hypothesis of EAE-induced lymphangiogenic cpLECs displaying a unique gene signature compared to steady state cpLECs. We next looked to further characterize the differences between the two groups, which may reveal novel functions of cpLECs in addition to their canonical roles in drainage. Since Cluster 2 consists primarily of EAE cpLECs, we decided to focus on the genes enriched within this cluster relative to the other groups. The gene enrichment pathways were divided into four main categories: 1) antigen processing and presentation (Figure 1G), 2) response to IFN- $\gamma$ (Figure 1H), 3) leukocyte adhesion/chemotaxis (Figure 1I), and 4) regulation of leukocyte activation (Figure 1J), in which six representative genes associated with these pathways are shown (Figure 1G - J). For each cluster, representative Cnet plots highlight the steady-state pathways in cluster 1, leukocyte crosstalk pathways in cluster 2, and proliferation pathways in cluster 3 along with all the associated genes and their relative strength of contribution to each pathway (Supplementary Figure 3B, D, F). These data demonstrate that cpLECs may not only mediate the drainage of leukocytes and antigens, but may also directly interact and engage in crosstalk with leukocytes through antigen processing/presentation, leukocyte adhesion/chemotaxis, and regulation of leukocyte activation, where some of these phenotypes may be acquired in response to IFN-Y signaling as a consequence of EAE. 


\section{Visualizing cpLEC trajectories.}

Since both healthy and EAE cells were almost exclusively clustered within clusters 1 and 2 with mixed cells in cluster 3 (Figure 1), we next asked if a healthy cell from cluster 1 can directly transition into an EAE-like cell in cluster 2 , or if it must first undergo a proliferative state like in cluster 3. Specifically, we asked if proliferation-driven lymphangiogenesis can account for all the lymphangiogenic cells in cluster 2, or if there may be some other mechanism that can account for the increased number of cpLECs during EAE. Indeed, there is evidence that inflammation-driven lymphangiogenesis may be driven through trans-differentiation of myeloid cells into LECs ${ }^{28-30}$. In line with this, we have previously demonstrated significant accumulation of myeloid cells in this region ${ }^{4}$. To investigate this, we visualized single-cell trajectories through pseudotime as previously described ${ }^{31-33}$, which allows us to visualize cell transitions from one state to another and consequently can reveal how far a cell has moved through a biological process. Visualization of cell trajectories through pseudotime revealed that a steady-state cell in cluster 1 seems to transition into a proliferative state in cluster 3 on its way to become an EAE-like state in cluster 2 (Supplementary Figure 4A). Visualizing specific genes revealed an upregulation of adhesion/chemotaxis genes such as CCL12, CCL2, CCL5, and CCL7 (Supplementary Figure 4B); IFN-Y response genes such as Ifitm3, Gbp2, and Stat1 (Supplementary Figure 4C); and antigen processing and presentation genes such as H2-Aa, H2-Q7, and Tap1 (Supplementary Figure 4D) through pseudotime relatively early, while genes involved in leukocyte activation such as Parp14, Slamf8, and CD274 (PD-L1) (Supplementary Figure 4E) seemed to be upregulated slightly later through pseudotime. These data suggest that lymphangiogenesis through the proliferation of pre-existing LECs seems to drive many of the phenotypic changes of cpLECs during EAE. Additionally, the proliferation of pre-existing lymphangiogenic vessels seem to account for all the EAE-like cells in cluster 2. Of note however, scRNA-seq revealed expression of many myeloid-associated genes by cpLECs in addition to LEC genes despite exclusion of CD45 $5^{\text {intermediate }}$ microglia and CD45 $5^{\text {high }}$ leukocytes (Supplementary Figure 1, 2) at the protein level while FACS sorting. Therefore, while proliferation of pre-existing LECs seem to account for all the lymphangiogenic cpLECs during EAE, we cannot exclude the involvement of myeloid cells in the origin of LECs as previously described and reviewed ${ }^{34,35}$.

\section{Neuroinflammation in EAE increases leukocyte binding to cpLECs.}


Conventionally, lymphatic endothelial cells have the ability to indirectly regulate immunity by facilitating leukocyte trafficking and drainage ${ }^{36,37}$. However, recent evidence has also suggested that lymphatic endothelial cells in the lymph nodes can directly regulate immunity through leukocyte crosstalk via ligands such as PD-L1 and/or antigen presentation ${ }^{19,22-25}$. Here, we show that cpLECs upstream of the lymph nodes and more local to the CNS are enriched for genes involved in antigen processing/presentation, leukocyte adhesion/chemotaxis, and regulation of leukocyte activation during EAE. Using cell-cell interaction analysis by flow cytometry ${ }^{38,39}$, we compared healthy and EAE cell interactions by gating on live doublets that express both the pan-leukocyte marker CD45 and cpLEC markers CD31, Podoplanin, and

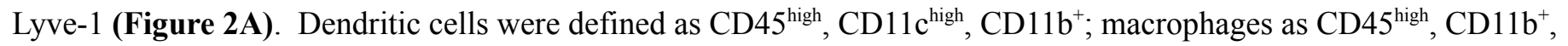

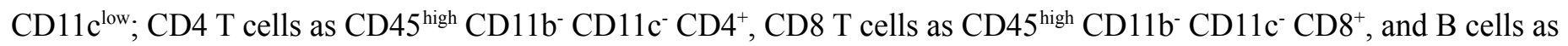

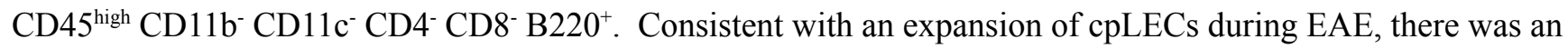
approximately 60 -fold increase in cpLEC cell number bound to $\mathrm{CD} 45^{+}$leukocytes, suggesting an increased affinity for cell binding by cpLECs during EAE (Figure 2B). Of the leukocytes, a significant increase in the number of CD $11 \mathrm{c}^{\text {high }}$ $\mathrm{CD}_{11 \mathrm{~b}^{+}}$dendritic cells $(\approx 130$-fold higher than steady-state $)$ and to a lesser extent $\mathrm{CD}^{+} \mathrm{T}$ cells $(\approx 16$-fold higher than steady-state) were bound to cpLECs, with a trending but non-significant increase in cpLEC bound $\mathrm{CD}_{11} \mathrm{~b}^{+} \mathrm{CD} 11 \mathrm{c}^{\text {low }}$ macrophages (Figure 2B). This data is consistent with the role of dendritic cells as professional antigen-presenting cells that can migrate to the lymphatics from the tissue parenchyma and validates our previous study demonstrating a large accumulation of inflammatory dendritic cells near the cribriform plate that contribute to the VEGFC-VEGFR3 dependent lymphangiogenesis of $\mathrm{cpLECs}^{4}$. No elevation was seen in the number of $\mathrm{CD} 8^{+} \mathrm{T}$ cells or $\mathrm{B} 220^{+} \mathrm{B}$ cells that were bound to cpLECs during EAE (Figure 2B). Of the leukocytes that bind to cpLECs, CD11 $\mathrm{c}^{\text {high }} \mathrm{CD} 11 \mathrm{~b}^{+}$dendritic cells made up the dominant cell type bound to cpLECs, with CD4 T cells binding to a lesser extent. The abundance of dendritic cells bound to cpLECs is consistent with their role as migratory cells that traffic to the lymph node through lymphatics (Figure 2C). We excluded the possibility of blood-derived leukocytes binding to cpLECs either in vivo through direct access between blood and lymphatic vessels, or ex vivo through blood-contamination by intravenously (IV) labeling blood-derived leukocytes with CD45.2 conjugated to BV711 in vivo, 3 minutes before harvest (Supplementary Figure 5). After perfusion, the majority of leukocytes binding to cpLECs were not blood-derived $(\approx 96 \%)$ (Supplementary Figure 5), suggesting that the leukocytes binding to cpLECs came from the brain parenchyma. In order to further validate our flow cytometry data, we induced EAE in CD11c-eYFP transgenic reporter mice and harvested cpLECs for visualization with confocal microscopy using the same harvest process for flow cytometry and stained for cpLECs using 
CD31 and Podoplanin. Doublets in the cell suspension consisted primarily of a CD11c-eYFP ${ }^{+}$cell bound to a CD31 ${ }^{+}$ Podoplanin $^{+}$cell (Figure 2D). In some cases, CD31 was expressed by both CD11c-eYFP ${ }^{+}$cells and Podoplanin ${ }^{+}$LECs

(Figure 2D), consistent with its function as a homophilic adhesion molecule ${ }^{40}$. We then validated our ex vivo cpLEC-leukocyte cell binding data using coronal sections of EAE CD11c-eYFP transgenic reporter mice to visualize dendritic cell-LEC binding in situ. IMARIS 3D surface volume rendering identified several CD11c-eYFP ${ }^{+}$cells in contact with cpLECs during EAE (Figure 2E), consistent with our previous study ${ }^{4}$. These data confirm that in response to neuroinflammation, $\mathrm{CD} 11 \mathrm{c}^{\text {high }} \mathrm{CD} 11 \mathrm{~b}^{+}$dendritic cells and, to a lesser extent $\mathrm{CD} 4^{+} \mathrm{T}$ cells, functionally bind to and interact with cpLECs during EAE.

\section{CpLECs can capture CNS-derived antigens and engage in antigen presentation with CD4 T cells during EAE.}

Of the gene pathways involved in cpLEC-leukocyte crosstalk, antigen processing and presentation was the most significant according to the scRNA-seq dataset, which correlates with our flow cytometry data showing an increased number of $\mathrm{CD} 11 \mathrm{c}^{\text {high }} \mathrm{CD} 11 \mathrm{~b}^{+}$dendritic cells and $\mathrm{CD}^{+} \mathrm{T}$ cell binding to cpLECs to potentially engage in crosstalk. Therefore, we next looked to see if cpLECs have access to CNS-derived antigens, if they can pick-up CNS-derived antigens, and if they can express MHC II. We induced EAE in CNP-Cre x OVA ${ }^{\mathrm{f} / \mathrm{fl}}(\mathrm{CNP}-\mathrm{OVA})$ transgenic mice, in which $\mathrm{OVA}_{257-264}-\mathrm{OVA}_{323-339}-\mathrm{GFP}$ is endogenously expressed under the CNPase promoter to drive expression by CNS oligodendrocytes as previously described ${ }^{41}$. These transgenic mice contain $\mathrm{OVA}_{257-264}-\mathrm{OVA}_{323-339}-\mathrm{GFP}$ within oligodendrocytes, which can functionally contribute to the proliferation of OVA-specific T cells in the draining lymph nodes during neuroinflammation ${ }^{4,41,42}$. Importantly, these mice allow us to investigate if cpLECs can gain access to CNS-derived antigens without disrupting the arachnoid barrier through cisterna magna or intracerebral injection of exogenous peptide. Immunolabeling of the cortex in CNP-OVA transgenic mice with antibodies against GFP and CNPase confirms expression of OVA-GFP within oligodendrocytes of the CNS (Figure 3A-C). During EAE, OVA-GFP ${ }^{+}$ expression could be observed not only near cpLECs, but also within Podoplanin ${ }^{+} \mathrm{MHC} \mathrm{II}^{+}$cpLECs (Figure 3D-H), suggesting cpLECs can access, acquire, and potentially present CNS-derived antigens through MHC II during EAE. Plot profile intensity analysis of cpLECs and nearby non-cpLECs reveal that a subpopulation of cpLECs contain OVA-GFP and express MHC II (Figure 3I). Control immunolabeling of the cribriform plate without anti-GFP antibody confirmed the lack of unspecific GFP signal in this region (Figure 3J-L). The expression of MHC II by cpLECs during EAE can also be validated by flow cytometry, where steady-state cpLECs contain much lower, if any, MHC II expression (Figure 
3M). Furthermore, a large accumulation of $\mathrm{CD} 11 \mathrm{c}^{\text {high }} \mathrm{MHC} \mathrm{II}^{+}$dendritic cells containing intracellular OVA-GFP can be observed within and near cpLECs during EAE (Figure 3N-R), suggesting that at least some of the CNS-derived antigens may be transported to cpLECs within dendritic cells. MHC II expression by cpLECs containing intracellular OVA-GFP also correlated with a large accumulation of CD4 T cells near cpLECs in situ, confirming our previous data of CD4 T cell binding with MHC II ${ }^{+}$cpLECs during EAE (Figure 3S- $\mathbf{U}_{\text {ii }}$ ). Taken together, these data suggest that lymphangiogenic cpLECs have the ability of capturing CNS-derived antigens, express MHC II, and functionally bind to dendritic and CD4 T cells during neuroinflammation.

To functionally test if cpLECs have the ability to process and present antigen to T cells, we generated a cpLEC-2D2 $\mathrm{T}$ cell co-culture to measure the ability of cpLECs to activate $\mathrm{MOG}_{35-55}$ specific $2 \mathrm{D} 2 \mathrm{~T}$ cells by measuring the early T cell activation marker CD69 after 24 hours of co-culture (Figure 3V-X). cpLECs from healthy or EAE-induced wild-type C57BL/6 mice were FACS sorted and co-cultured with magnetically purified 2D2 T cells from splenocytes of naive 2D2 T cell receptor transgenic mice using CD4 negative selection. Healthy cpLECs induce negligible levels of T cell activation (approximately 6\% of 2D2 T cells expressing CD69) (Figure 3V, X). In contrast, cpLECs from EAE mice are able to significantly induce CD69 expression in approximately 43\% of 2D2 T cells (Figure 3W, X), suggesting that cpLECs are able to engage in antigen processing, presentation, and consequently $\mathrm{T}$ cell activation at much higher levels during EAE. Furthermore, after 72 hours of co-culture healthy cpLECs were only able to induce T cell proliferation in approximately 22\% of 2D2 T cells (Figure 3Y, $\mathbf{A}_{\mathbf{a}}$ ), while EAE-primed cpLECs induced T cell proliferation in approximately 38\% of 2D2 T cells (Figure 3Z, $\mathbf{A}_{\mathbf{a}}$ ), reflecting a 1.7 fold increase in T cell proliferation with many of these proliferated T cells undergoing additional cycles of proliferation (Figure 3Y-A $\mathbf{A}_{\mathbf{a}}$ ). Additionally, EAE-primed cpLECs can also functionally present another antigenic peptide, $\mathrm{OVA}_{323-339}$, to activate $\mathrm{OVA}_{323-339}$-specific OT-II CD4 T cells using the same co-culture model when cpLECs were isolated from EAE-induced CNP-OVA transgenic mice as described (Supplementary Figure 6). We focussed on OVA $_{323-339}$-specific OT-II T cells due to the fact that this particular model of EAE elicits a primarily CD4 T cell response, with no significant levels of CD8 T cell interaction with cpLECs during EAE (Figure 2B). These data show that unlike steady-state lymphatics, EAE-primed cpLECs are able to undergo lymphangiogenesis to facilitate drainage ${ }^{4}$ as well as functionally process and present antigens to CD4 T cells through MHC II during EAE.

Neuroinflammation increases cpLEC expression of proteins involved in leukocyte binding and crosstalk. 
While characterizing cpLECs by flow cytometry, we noticed that many of the markers used to identify lymphatic endothelial cells were up-regulated at the protein level during neuroinflammation. Many of these markers, including Podoplanin, Lyve-1, and CD31 are all implicated in lymphatic vessel-leukocyte interactions ${ }^{43-46}$, consistent with our previous data showing increased cpLEC-leukocyte binding (Figure 2). While scRNA-seq was unable to screen for these particular markers with sufficient average counts, other genes such as PD-L1 (CD274), a tolerogenic ligand that can interact with the PD-1 receptor expressed by dendritic cells and T cells during inflammation ${ }^{19,22-24}$, was also enriched by cpLECs during EAE (Figure 1). In order to investigate if these same markers correlated with dendritic cell and CD4 T cell binding to cpLECs, we validated Podoplanin, Lyve-1, CD31, and PD-L1 upregulation by cpLECs during EAE at the protein level using flow cytometry (Supplementary Figure 7). When gating for live, singlet cpLECs (Supplementary Figure 7A), the expression of Podoplanin (Supplementary Figure 7B - E), Lyve-1 (Supplementary Figure 7F - I), CD31 (Supplementary Figure 7J - M), and PD-L1 (Supplementary Figure 7N - Q) were indeed up-regulated during EAE compared to healthy controls. Of note, the expression of these proteins were further upregulated when gating for cpLEC-leukocyte doublets with the exception of Lyve-1, which may suggest that leukocytes preferably bind to cpLECs with the highest expression of these proteins. Alternatively, CD31 ${ }^{43}$ and PD-L1 ${ }^{22}$ have also been reported to be expressed by leukocytes/dendritic cells respectively, which may increase the doublet MFI when bound to a cpLEC that also expresses these markers. While background median fluorescence intensity (MFI) when gating for doublets versus singlets could be observed for some markers, the increase in MFI due to doublet background was negligible when compared to actual protein expression (Supplementary Figure 8). Increased cpLEC expression of Podoplanin, Lyve-1, CD31, and PD-L1 during EAE suggests that cpLECs functionally alter their phenotype to promote leukocyte adhesion/chemotaxis through Podoplanin, Lyve-1, and CD31, as well as engage in cross talk through PD-L1.

\section{Upregulation of Podoplanin and PD-L1 by cpLECs is mediated by IFN-Y.}

We next investigated what potential cytokine may be involved in upregulation of leukocyte-interacting proteins by cpLECs during EAE. EAE induced in C57BL/6 mice are characterized by a strong Th1/Th17 response ${ }^{47}$. IFN-Y has also been hypothesized to regulate both Podoplanin and PD-L1 expression by peripheral lymphatic vessels during inflammation ${ }^{48,49}$. Coincidentally, our scRNA-seq data shows an enrichment in IFN-y response genes by cpLECs during

EAE (Figure 1), which coincides with PD-L1 upregulation, an immune checkpoint protein (Figure 1, Supplementary Figure 7). Furthermore, genes involved in response to IFN-Y by cpLECs appeared earlier in pseudotime compared to 
genes involved in leukocyte activation such as PD-L1 (Supplementary Figure 4), suggesting that IFN-Y may regulate the expression of PD-L1. Mice deficient in the pro-inflammatory cytokine IFN- $\gamma$ have exacerbated EAE ${ }^{50,51}$, suggesting a tolerogenic role for IFN-ץ. Thus, we hypothesized that IFN-Y may be responsible for the increased expression of Podoplanin and PD-L1 by cpLECs (Supplementary Figure 7), which may partially explain the tolerogenic role of IFN- $\gamma$ during EAE. EAE was induced in wild-type and IFN- $\mathrm{Y}^{-/ 2}$ mice, and at EAE score 3.0 both strains were analyzed for the expression of Podoplanin and PD-L1 to compare to healthy wild-type controls (Figure 4A - I). Identically scored EAE mice were used to prevent any effects due to differences in EAE severity between wild-type and IFN- $\gamma^{-/ 2}$ mice, since IFN- $\gamma^{-/-}$mice have exacerbated EAE severity ${ }^{50,51}$. Similar to our previous results, EAE upregulates Podoplanin, PD-L1, and CD31 expression by cpLECs, as well as PD-L1 expression by dendritic cells (Figure 4B - I). CpLECs from the IFN- $\gamma^{-/}$mice have reduced expression of Podoplanin and PD-L1 while CD31 expression remained unchanged, suggesting Podoplanin and PD-L1 up-regulation during EAE are both dependent on IFN-Y signaling (Figure 4B - I).

Since IFN-Y seemed to regulate some of the phenotypes of EAE-induced lymphangiogenic cpLECs, we next looked to see if IFN- $\gamma$ is required for lymphangiogenesis. To test this, we gated for cpLECs to measure lymphangiogenesis between healthy wild-type mice, EAE wild-type mice, and EAE IFN- $\gamma^{-/-}$mice. CpLECs from IFN- $\gamma^{-/-}$ mice still underwent lymphangiogenesis during EAE (Supplementary Figure 9), suggesting that although lymphangiogenesis does not require IFN-Y signaling, some of the acquired phenotypes of lymphangiogenic vessels including Podoplanin and PD-L1 upregulation does depend onIFN- $\gamma$ signaling. Taken together, these data suggest IFN-Y can regulate some of the phenotypes and functions of cpLECs during EAE.

\section{CpLECs are in a prime position to access the CSF compartment.}

The ability of cpLECs to undergo lymphangiogenesis to facilitate excess drainage of fluid occurs under the assumption that they should have direct access to the CSF-filled subarachnoid space. We and others have previously demonstrated that the different meningeal lymphatics can access $\mathrm{CSF}^{1-4}$, yet how they access the CSF-filled subarachnoid space through the E-Cadherin ${ }^{+}$epithelial cells that make up the arachnoid barrier is unknown ${ }^{5}$. A previous study has identified gaps in the arachnoid barrier of developing rats, suggesting direct access between cpLECs and the subarachnoid space $^{52}$. Furthermore, the ability of cpLECs to uniquely alter their phenotype and undergo lymphangiogenesis during EAE suggests they may have unique access to the CNS microenvironment. In order to visualize the epithelial cells that 
make up the arachnoid barrier separating the dural LECs above the sinuses, meningeal LECs at the base of the brain, and cpLECs from the subarachnoid space, we immunolabeled coronal sections with the epithelial adherens junction protein E-Cadherin (Figure 5A-C). Our data validates Ahn et al.'s study in which there is a continuous, uninterrupted E-Cadherin $^{+}$arachnoid barrier separating both the dural LECs and basal meningeal LECs from the subarachnoid space, with basal meningeal LECs residing closer in proximity to the subarachnoid space ${ }^{3}$ (Figure $\mathbf{5 B}-\mathbf{C}$ ). In contrast, here we show that there are several large gaps in E-Cadherin labeling between cpLECs and the subarachnoid space (Figure 5A), which has only been previously seen in developing rats ${ }^{52}$. These data suggest that lymphatics near the cribriform plate may have direct access to the CSF-filled subarachnoid space through gaps in the arachnoid barrier.

Since cpLECs seem to have direct access to the CSF-filled subarachnoid space through gaps in the arachnoid barrier, we next looked to see if cpLECs can express proteins involved in fluid uptake and transport such as Aquaporin-1 (AQP-1) ${ }^{53}$. Immunohistochemistry of the cribriform plate reveals the expression of AQP-1 by cpLECs, highlighting a potential mechanism of fluid transport from the subarachnoid space into cpLECs (Figure 5D - G). Additionally, AQP-1 is expressed throughout the lymphatic vessel, even after lymphangiogenic expansion of cpLECs during EAE (Figure 5D, F). These data suggest that there may be compensatory mechanisms to manage neuroinflammation-induced edema with increased AQP-1 expression by lymphangiogenic cpLECs. Both the gaps in the arachnoid barrier and expression of the water pore AQP-1 by cpLECs highlight their ability to sample the CSF compartment, and may explain their unique ability to undergo lymphangiogenesis during neuroinflammation.

In addition to CSF access, we visualized CSF dynamics using Magnetic Resonance Imaging (MRI) before and after Gadolinium injection into the cisterna magna (Figure 6A-E) to determine if cpLECs can sample from a large CSF reservoir. Similar to a previous report, the majority of Gadolinium accumulation over time occurred at the base of the brain near basal $\mathrm{mLVs}^{3}$ (Figure 6B). However, we also found a relatively equal amount of Gadolinium accumulation at the base of the olfactory bulbs near the cribriform plate, suggesting that cpLECs have access to a reservoir of CSF (Figure 6B). Additionally, our data revealed a relative increase in Gadolinium accumulation near both the base of the brain and cribriform plate during EAE, consistent with the inflammation-induced edema (Figure 6B, D). Consequently, there was an increase in Gadolinium accumulation in the deep cervical lymph nodes (Figure 6C, E) downstream of CNS lymphatics, which correlates with functional lymphangiogenesis by cpLECs. This data not only suggest cpLECs have direct access to a CSF reservoir, but that lymphangiogenesis potentially occurs in response to neuroinflammation to manage edema. 


\section{Discussion}

Unlike lymph node resident LECs in which a subpopulation of LECs can directly engage in leukocyte crosstalk and regulation through antigen presentation and ligands such as PD-L1 during steady-state conditions ${ }^{17-21}$, meningeal lymphatics do not have this capability during homeostasis ${ }^{54}$ (Figure 1). Stromal cells in the meninges such as resident macrophages and dendritic cells can directly sample antigen from the CSF compartment and contribute to immune surveillance during steady-state conditions, highlighting the meninges as a neuro-immune interface. In this study, we provide data that neuroinflammation can prime cpLECs to undergo lymphangiogenesis and acquire a unique phenotypic signature to engage in leukocyte crosstalk and regulation, similarly to lymph node resident LECs. This suggests that during neuroinflammation, cpLECs dynamically respond to cues from the CNS microenvironment to alter their phenotype. These cues induce a variety of changes by LECs including lymphangiogenesis driven by recruited VEGFC-producing myeloid cells ${ }^{4}$, upregulation of leukocyte crosstalk and regulation proteins such as Podoplanin and PD-L1 through IFN-Y (Figure 4), as well as the antigen processing and presentation machinery (Figure 3). These changes by cpLECs can then in turn regulate leukocytes that bind and interact to cpLECs during EAE, consisting primarily of dendritic cells and to a lesser extent CD4 T cells (Figure 2). Migratory professional antigen presenting dendritic cells have been shown to traffick to the draining lymph nodes through lymphatic vessels during neuroinflammation through a variety of signaling mechanisms such as CCR7/CCL2 $1^{55,56}$, Podoplanin/Clec2 ${ }^{44,57}$, Vcam-1 ${ }^{58}, \mathrm{CD} 31^{46}$, and Lyve-1 ${ }^{45,59}$. Here we show that cpLECs not only facilitate the drainage of leukocytes, but functionally bind to dendritic cells and CD4 T cells to engage in cross talk. Dendritic cells can also express the PD-L1 receptor PD-1 ${ }^{60-64}$, suggesting that some of the dendritic cells bound to cpLECs may become tolerized. In addition to dendritic cells, CD4 T cells to a lesser extent also bind and interact with EAE-primed cpLECs. We demonstrate that cpLECs can capture CNS-derived antigens and functionally present antigen to activate antigen specific CD4 T cells during EAE.

Our scRNA-seq dataset of cpLECs revealed an upregulation of genes involved in the IFN-Y response, one of the highest-enriched cytokines in the CNS during EAE ${ }^{65}$. Interestingly IFN-Y is not required for lymphangiogenesis (Supplementary Figure 9), but is required for some of the phenotypes acquired by lymphangiogenic vessels during EAE such as the upregulation of PD-L1 and Podoplanin. EAE is exacerbated in mice that lack either IFN-Y or IFN- $\gamma$ receptor ${ }^{50}$, suggesting a tolerogenic role for IFN-ץ in CNS autoimmunity which coincides with the IFN- $\gamma$-dependent upregulation of PD-L1 by cpLECs. IFN-Y is also required for dendritic cell expression of PD-L1 during EAE, and depletion of dendritic cells after the onset of EAE exacerbates EAE severity ${ }^{66}$, suggesting that both cpLECs and dendritic cells may 
play an IFN- $\gamma$-dependent tolerogenic role in the later phase of EAE. The role of IFN- $\gamma$ in EAE is diverse and stage-specific ${ }^{51}$, and the tolerogenic role of IFN-Y in the later phases likely involves a variety of mechanisms including the accumulation of myelin antigens within the CNS due to dysfunctional APCs ${ }^{50}$.

Lymphangiogenesis often accompanies inflammation, however lymphangiogenesis of meningeal lymphatic seems to be context-dependent. Meningeal lymphatics near the cribriform plate can undergo lymphangiogenesis during EAE ${ }^{4,6}$, however, lymphangiogenesis could not be observed by dural lymphatics above the sinuses ${ }^{4,6}$. This highlights the potential differences in the meningeal lymphatic network microenvironments, areas of the CNS they may sample, and how they may respond to different diseases. Bulk RNA sequencing of dural lymphatics have highlighted dysregulation of genes involved in extracellular matrix, focal adhesion, angiogenesis, responses to endogenous and external stimuli, development, proliferation, and stiffness related genes, suggesting that dural lymphatics may be limited in their ability to undergo lymphangiogenesis ${ }^{6}$. Nevertheless, lymphangiogenesis by dural lymphatics above the sinuses can be observed in models where neuroinflammation is local enough to the dura mater such as during traumatic brain injury, chronically implanted EEGs, and in certain models of intracranial tumors ${ }^{8,16,67}$. Interestingly, lymphangiogenic vessels in the dura above the sinuses can be observed in a superficial brain tumor model where tumor cells are injected subdurally. Bulk RNA sequencing from this study also revealed an enrichment in antigen processing and presentation through MHC II among other similar immune related genes ${ }^{8}$. Although this tumor study did not provide functional evidence for leukocyte crosstalk and regulation by meningeal LECs, together with our data these studies suggests that although there is heterogeneity in the ability of meningeal lymphatics to undergo lymphangiogenesis, the ability of lymphangiogenic vessels to engage in leukocyte crosstalk and directly regulate leukocyte function as non-professional antigen presenting cells during neuroinflammation may be shared across the different meningeal lymphatic networks. Sequencing studies of the dural tissue have suggested that dural lymphatics lack the ability to engage in antigen processing and presentation during steady-state conditions ${ }^{54}$, similar to cpLECs during homeostasis (Figure 1), suggesting that the meningeal lymphatics may require additional cues during neuroinflammation to acquire the antigen processing and presentation machinery.

Previously we have shown that neuroinflammation recruits VEGFC-producing myeloid cells to drive meningeal lymphangiogenesis near the cribriform plate to facilitate excess drainage of fluid, cells, and antigens to promote $\mathrm{T}$ cell priming in the lymph node ${ }^{4}$. Here we show that lymphatics near the cribriform plate can acquire an altered phenotype during neuroinflammation that facilitates dendritic cell and CD4 T cell retention for leukocyte crosstalk and regulation 
through the upregulation of adhesion molecules, some of which are regulated by IFN-ץ. The crosstalk between dendritic cells, cpLECs, and CD4 T cells involve antigen transfer, presentation, and regulation of molecules such as MHC II and PD-L1, highlighting a previously underappreciated interface of meningeal lymphatics near the cribriform plate as an immune-regulatory niche.

\section{Acknowledgements}

We thank Khen Maclivay for his expertise in flow cytometry, Laura Schmitt-Brunold for her expertise in molecular biology, and all members of our laboratory for insightful discussions and constructive criticisms of this work. We would like to thank members of the UW Flow Core Facility for their assistance and expertise in FACS sorting, Tyler Duellman and Sandra Splinter BonDurant for their expertise and assistance in scRNA-seq, as well as Beth Rauch and Elizabeth Meyerand at the UW Small Animal Imaging Facility (SAIF) supported by the UWCCC grant P30 CA014520 for use of its facilities and services. This work was supported by National Institutes of Health grants NS10847 and NS103506 awarded to Z.F., HL128778 awarded to M.S., the Neuroscience Training Program T32-GM007507 to M.H. and C.L., and the 10X Genomics Pilot award grant to Z.F.

\section{Author Contributions}

M.H. ${ }^{1}$, M.S., and Z.F. conceptualized the experiments. M.H. ${ }^{1}$ performed the experiments, generated the figures, analyzed the data, and wrote the manuscript. A.M. performed the analysis and assisted in generating figures for the scRNA-seq data. C.L. assisted with the FACS for scRNA-seq. C.L. and M.H. ${ }^{2}$ performed the I.V. CD45 timing experiments, and assisted with the in vitro co-culture experiments. Y.H.C. assisted with the MRI experiments. All authors edited the manuscript. 


\section{Materials and Methods}

\section{Animals}

Female C57BL/6J wild-type (stock \#: 000664), IFN- $\gamma^{-/ 2}$ mice (stock \#: 002287), 2D2 TCR transgenic mice (stock \#: 006912), and OT-II TCR transgenic mice (stock \#: 004194) were purchased from Jackson Laboratories. CD11c-eYFP transgenic reporter mice were a generous gift from Dr. Michel C. Nussenzweig at Rockefeller University. CNP-Cre transgenic mice were a generous gift from Brian Popco at the University of Chicago. pZ/EG-OP OVA $257-264-\mathrm{OVA}_{323-339}$ $\left(\mathrm{OVA}^{\mathrm{f} / \mathrm{fl}}\right.$ mice) were generated by our lab as previously described in the $\mathrm{C} 57 \mathrm{BL} / 6$ background ${ }^{41}$. OVA ${ }^{\mathrm{fl} / \mathrm{fl}}$ mice were crossed with CNP-Cre mice to generate CNP-OVA transgenic mice that express $\mathrm{OVA}_{257-264}$ and $\mathrm{OVA}_{323-339}$ with GFP under the CNS oligodendrocyte-specific CNPase promoter ${ }^{4,41}$. Eight to twelve-week old female mice were used for all EAE experiments along with the appropriate age and sex matched controls. All experiments were conducted in accordance with guidelines from the National Institutes of Health and the University of Wisconsin - Madison Institutional Animal Care and Use Committee.

\section{EAE Induction}

EAE was induced in 8 to 12 -week-old female mice by subcutaneous immunization with 100 ug of $\mathrm{MOG}_{35-55}$ emulsified in Complete Fruend's Adjuvant (CFA) between the shoulder blades. 200 ng of Pertussis Toxin (PTX) was injected intraperitoneally at 0 days post immunization (d.p.i.) and 2 d.p.i. The onset of clinical scores were observed between day 8 and 12 post-immunization, and were assessed daily as follows: 0 , no clinical symptoms; 1, limp/flaccid tail; 2, partial hind limb paralysis; 3, complete hind limb paralysis; 4, quadriplegia; 5, moribund. Intermediate scores were also given for the appropriate symptoms.

\section{Magnetic Resonance Imaging.}

MRI experiments were done using a 4.7T small animal MRI (Agilent Technologies Inc., Santa Clara, CA, USA) and acquired using VnmrJ (Agilent Technologies). After scout scans, isotropic 3D T1-weighted scans were used to detect gadolinium using the following parameters: $\mathrm{TR}=9.3 \mathrm{~ms}$; $\mathrm{TE}=4.7 \mathrm{~ms}$; Flip Angle $=20$ degrees; Field of View $=$ 40x20x20 mm; Resolution $=256 \times 128 \times 128$; Averages $=4$; Voxel Size $» 156 \mu \mathrm{m}^{3}$. These resulted in a time scan of approximately 11 minutes. Animals were anesthetized using isoflurane administered through a nose-cone, and $10 \mu 1$ of gadolinium was injected into the cisterna magna at a rate of $2 \mu 1 /$ minute. Respiratory rates and body temperature were 
monitored to ensure normal physiology. A baseline scan was acquired prior to gadolinium injection, and image processing was done using FIJI software.

\section{Histology.}

Mice were terminally anesthetized with isoflurane and transcardially perfused with $0.1 \mathrm{M}$ PBS followed by perfusion with $4 \%$ paraformaldehyde (PFA) in $0.1 \mathrm{M}$ PBS. Mice were then decapitated and the skin surrounding the head was removed using forceps and scissors to separate the skin from the muscle and ear canal. The whole heads were fixed in $4 \%$ PFA in $0.1 \mathrm{M}$ PBS overnight. The whole heads were then decalcified in $14 \%$ ethylenediaminetetraacetic acid (EDTA) in $0.1 \mathrm{M}$ PBS for 7 days followed by cryoprotection in $30 \%$ sucrose in $0.1 \mathrm{M}$ PBS for 3 days. The EDTA was replaced with fresh 14\% EDTA each day. The decalcified mouse heads were then embedded in Tissue-Tek OCT Compound, frozen on dry ice, then stored at $-80^{\circ} \mathrm{C}$. $60 \mu \mathrm{m}$ thick frozen sections were obtained on a Leica CM1800 cryostat, mounted on Superfrost Plus microscope slides and stored at $-80^{\circ} \mathrm{C}$. For dural tissues, the skullcap was isolated and collected after decalcification and stored at $4^{\circ} \mathrm{C}$ in a 48 -well non-tissue culture treated plate containing PBS.

\section{Immunohistochemistry}

Sections were thawed at room temperature for 10 minutes, washed with $0.1 \mathrm{M}$ PBS for 10 minutes, then unspecific binding blocked with $10 \%$ bovine serum albumin (BSA) with $0.1 \%$ Triton-X for permeabilization in $0.1 \mathrm{M}$ PBS for 60 minutes. Sections were then incubated with the appropriate primary antibodies in $1 \% \mathrm{BSA}$ and $0.1 \%$ Triton-X in $0.1 \mathrm{M}$ PBS at $4^{\circ} \mathrm{C}$ overnight in a humidified chamber. The following antibodies were used for immunohistochemistry:

Podoplanin PE (eBioscience, Catalog \#: 12-5381-80), CD31 Alexa647 (BD Biosciences, Catalog \#: 563608), Lyve-1 eFluor660 (Thermo Fisher Scientific, Catalog \#: 50-0443-80), rabbit anti-GFP unconjugated (Novus Biologicals, Catalog \#: NB100-1614-0.02ml), CNPase Alexa647 (Biolegend, Catalog \#: 836407), MHC II eFluor450 (eBioscience, Catalog \#: 48-5321-80), CD11c Alexa488 (Thermo Fisher Scientific, Catalog \#: 53-0114-80), CD4 PE (BD Pharmingen, Catalog \#: 553049), Goat anti-E-Cadherin unconjugated (R\&D Systems, Catalog \#: AF748), and rabbit anti-AQP-1 unconjugated (EMD Millipore, Catalog \#: AB2219). Sections were then washed three times with 0.1M PBS for 10 minutes each, then incubated with the appropriate secondary antibodies in $1 \%$ BSA and $0.1 \%$ Triton-X in $0.1 \mathrm{M}$ PBS at room temperature for 120 minutes. The following secondary antibodies were used: Donkey anti-Chicken Alexa488 (Invitrogen, Catalog \#: 
Catalog \#: A11057), and/or Donkey anti-rabbit Alexa568 (Invitrogen, Catalog \#: A10042). Sections were then washed three times with $0.1 \mathrm{M}$ PBS for 10 minutes each, mounted with Prolong Gold mounting medium with DAPI, and images acquired using an inverted Olympus Fluoview FV1200 confocal microscope. For imaging of the dura, the whole dura attached to the skullcap underwent the same immunohistochemistry protocol above using a free-floating method in a 48-well plate. The whole skullcap with the dura attached was placed on a microscope slide and imaged using an inverted Olympus Fluoview FV1200 confocal microscope, with occasional application of PBS to prevent dehydration. The brightness/contrast of each image was applied equally across the entire image and equally across all images and analyzed using either FIJI or IMARIS software.

\section{Single Cell Suspension}

Mice were terminally anesthetized with isoflurane and transcardially perfused with PBS. Mice were then decapitated and the skin was cut dorsal to the midline of the skullcap rostrally to expose the skullcap. The skullcap was then removed along with the brain after separation from the olfactory bulbs. The cribriform plate and its associated tissues which included the olfactory bulbs, the cribriform plate, and parts of the nasal mucosa were dissected out and placed in a 70-micron strainer submerged in RPMI-1640 in a non-tissue culture treated dish. The tissues were then mechanically dissociated by pushing the tissue through the strainer using a syringe plunger. The mechanically dissociated cells were then spun down, washed, and resuspended in FACS buffer (1\% Bovine Serum Albumin in 0.1M PBS) for FACS staining.

\section{Flow Cytometry}

After resuspension of mechanically dissociated cells in fluorescence-activated cell sorting (FACS) buffer ( $\mathrm{pH}=$ 7.4, 0.1M PBS, $1 \mathrm{mM}$ EDTA, 1\% BSA), the cells underwent staining with conjugated antibodies/dyes. Conjugated antibodies were diluted 1:200 in FACS buffer, and the following antibodies and dyes were used: Ghost UV450 (Tonbo Biosciences, Catalog \#: 13-0868-T500) or Ghost Violet540 (Tonbo Biosciences, Catalog \#: 13-0879-T100) to visualize live/dead cells; CD31 Alexa647 (BD Biosciences, Catalog \#: 563608), Podoplanin PE (eBioscience, Catalog \#: 12-5381-82), and Lyve-1 Alexa488 (eBioscience, Catalog \#: 53-0443-82) to visualize lymphatic endothelial cells; CD45 APC-Cy7 (Biolegend, Catalog \#: 103116) or CD45 APC eFluor780 (eBioscience, Catalog \#: 47-0451-80) to visualize leukocytes; CD11b PerCP-Cy5.5 (Biolegend, Catalog \#: 101227) or CD11b PE-Cy5 (Biolegend, Catalog \#: 101210) to 
visualize myeloid cells; CD11c BV421 (Biolegend, Catalog \#: 117329) or CD11c FITC (Biolegend, Catalog \#: 117305) to visualize dendritic cells; CD8 PE-Cy7 to visualize cytotoxic T cells (Biolegend, Catalog \#: 100721); CD4 BUV496 to visualize T helper cells (BD Bioscience, Catalog \#: 564667); B220 BV510 to visualize B cells (Biolegend, Catalog \#: 103247); PD-L1 PE-Cy7 to visualize the tolerogenic ligand PD-L1 (Biolegend, Catalog \#: 124313). After staining for 30 minutes at $4^{\circ} \mathrm{C}$, cells were washed 3 times with FACS buffer and processed using BD LSR II or Cytek's 3-laser Northern Lights.

\section{FACS Sorting}

After generating a single cell suspension and staining for cpLECs with Ghost UV450 (Tonbo Biosciences, Catalog \#: 13-0868-T500), CD31 Alexa 647 (BD Biosciences, Catalog \#: 565608), Podoplanin PE (eBioscience, Catalog \#: 12-5381-82), and CD45 APC-Cy7 (Biolegend, Catalog \#: 103116), the cells were sorted with a FACS Aria III with a nozzle size of 130 um at the UW Flow Core satellite facility in the UW Biotechnology Center. LECs were gated for singlets using both FSC and SSC, Ghost ${ }^{\text {negative }}$ live cells, and both CD $31^{+}$Podoplanin $^{+}$after excluding both CD45 $5^{\text {intermediate }}$ microglia and $\mathrm{CD}_{4} 5^{+}$leukocytes as previously described ${ }^{2,8,10,26}$. Cells were sorted into either FACS buffer (1\% BSA in PBS) for scRNA-seq, or RPMI containing 10\% FBS, 2-mercaptoethanol, and penicillin/streptomycin for in vitro co-culture experiments with CD4 T cells to measure antigen presentation capability.

\section{In vitro $\mathrm{T}$ cell activation assay}

Healthy or EAE cpLECs were isolated and FACS sorted as previously described, and cultured in a 96-well flat bottom non-tissue culture treated plate in RPMI containing 10\% heat-inactivated fetal bovine serum (FBS), 2-mercaptoethanol, and penicillin/streptomycin. Splenocytes were generated from either naive 2D2 TCR transgenic mice for $\mathrm{MOG}_{35-55}$ specific 2D2 CD4 T cells, or from OT-II TCR transgenic mice for OVA $323-339$ specific OT-II CD4 T cells using a magnetic negative selection kit (Miltenyi, Catalog\#: 130-104-454) following the manufacturer's recommended protocol. Once purified, 2D2 or OT-II T cells were labeled with Cell Trace Violet (ThermoFisher Scientific, Catalog\#: C34557) following the manufacturer's recommended protocol, and co-cultured with cpLECs (100,000-200,000 CD4 T cells:5,000-10,000 cpLECs). After 24 hours the co-culture underwent FACS staining and analysis to measure the early T cell activation marker CD69 (Biolegend, Catalog\#: 104510), or after 72 hours T cell proliferation through the dilution of Cell Trace Violet. 


\section{In vivo CD45 I.V. labeling}

EAE was induced in C57BL/6 wild-type mice, and at peak EAE mice were intravenously (I.V.) injected with CD45.2 antibody conjugated to BV711 (Biolegend, Catalog \#: 109847) 3 minutes before harvest to control for ex vivo unspecific binding of blood-derived cells to LECs and/or to visualize direct in vivo access between blood vessels and lymphatic vessels. Unperfused mice were used as positive controls to visualize blood-derived binding of leukocytes to cpLECs. Perfused mice were used for all experiments. cpLECs were then processed for flow as described previously, and processed using Cytek’s 3-laser Northern Lights.

\section{scRNA Sequencing}

5 healthy mice were pooled for the control group, and 5 EAE mice were pooled for the experimental EAE group. A single cell suspension of the cribriform plate and its associated tissues were generated as described, and cpLECs were FACS sorted for singlets, Ghost live cells, CD45 ${ }^{\text {lo }}$, Podoplanin ${ }^{+}$, and CD31 $1^{+2,6,8,10,26}$. Sorted cpLECs were then provided to the Biotechnology Core facility at the University of Wisconsin Madison for single cell sequencing using the 10x Genomics Chromium Single Cell Gene Expression Assay. A total of 25,102 cells from the control group and 53,224 cells from the experimental group post-sort were provided to the Biotechnology Core for scRNA-seq. The target recovery rate was 3,000 cells with a targeted read depth of 7,500. Cells were loaded onto a Chromium Controller to generate a single cell + barcoded gel-bead emulsion from which Illumina-compatible library preps were generated and sequenced on the NovaSeq in collaboration with the University of Wisconsin Biotechnology Center (UWBC) DNA Sequencing Facility. Data was then analyzed using a custom developed single cell data analysis pipeline generated by the UWBC Bioinformatics Resource Center. The resulting data were then analyzed and explored using the Loupe Cell Browser software and various R packages after excluding non-LEC cells that made it through the FACS-enrichment.

\section{Gene ontologies}

Genes identified to be differentially expressed from each cluster were separately assessed by gene ontological analyses using R package clusterProfiler ${ }^{68}$. The genes that passed filtering during alignment were used as the background set during over-representation analyses. Significant gene ontological terms were identified using an adjusted P-value $<$ 0.05. Bar plots, gene-concept network plots, and enrichment maps were visualized and generated using clusterProfiler. 


\section{Single cell trajectory analysis}

R package monocle ${ }^{31-33}$ was employed for pseudotime analysis. Feature, gene annotation, and cell annotation matrices were regenerated for cells stemming from clusters of interest using het cellranger reanalyze function. A cell dataset was generated using these matrices and preprocessed using 50 as the maximum number of dimensions. Dimensions were reduced using UMAP methodology. Cells were clustered and trajectories were learned using default parameters by monocle3. Cells were ordered and pseudotime was calculated in reference to a node in the healthy cell cluster. Expression data was extracted for genes of interest and plotted against estimated pseudotime, using a minimum expression cutoff of 5 reads for visualization purposes.

\section{Statistics}

When comparing results from two groups, unpaired Student's t-test was used. When comparing results from three groups, one-way ANOVA using Tukey's post-hoc multiple comparisons test was used. When comparing results across time, two-way ANOVA using Sidak's multiple comparisons test was used. All statistical analysis was performed using Graphpad Prism 6.0 software. Data is portrayed as the mean $+/$ - standard error of the mean (S.E.M.), and significance portrayed as: n.s. $=$ non-significant, $p>0.05 ; * p<0.05 ; * * p<0.01 ; * * *<0.001 ; * * * * p<0.0001$. 


\section{References}

1. Aspelund, A. et al. A dural lymphatic vascular system that drains brain interstitial fluid and macromolecules. $J$. Exp. Med. 212, 991-999 (2015).

2. Louveau, A. et al. Structural and functional features of central nervous system lymphatic vessels. Nature vol. 523 $337-341(2015)$.

3. Ahn, J. H. et al. Meningeal lymphatic vessels at the skull base drain cerebrospinal fluid. Nature 572, $62-66$ (2019).

4. Hsu, M. et al. Neuroinflammation-induced lymphangiogenesis near the cribriform plate contributes to drainage of CNS-derived antigens and immune cells. Nat. Commun. 10, 229 (2019).

5. Proulx, S. T. Cerebrospinal fluid outflow: a review of the historical and contemporary evidence for arachnoid villi, perineural routes, and dural lymphatics. Cell. Mol. Life Sci. 78, 2429-2457 (2021).

6. Louveau, A. et al. CNS lymphatic drainage and neuroinflammation are regulated by meningeal lymphatic vasculature. Nat. Neurosci. 21, 1380-1391 (2018).

7. Song, E. et al. VEGF-C-driven lymphatic drainage enables immunosurveillance of brain tumours. Nature 577, 689-694 (2020).

8. Hu, X. et al. Meningeal lymphatic vessels regulate brain tumor drainage and immunity. Cell Res. 30, 229-243 (2020).

9. Ma, Q. et al. Lymphatic outflow of cerebrospinal fluid is reduced in glioma. Sci. Rep. 9, 14815 (2019).

10. Da Mesquita, S. et al. Functional aspects of meningeal lymphatics in ageing and Alzheimer's disease. Nature 560, 185-191 (2018).

11. Ma, Q., Ineichen, B. V., Detmar, M. \& Proulx, S. T. Outflow of cerebrospinal fluid is predominantly through lymphatic vessels and is reduced in aged mice. Nat. Commun. 8, 1434 (2017).

12. Ding, X.-B. et al. Impaired meningeal lymphatic drainage in patients with idiopathic Parkinson's disease. Nature Medicine vol. 27 411-418 (2021).

13. Esposito, E. et al. Brain-to-cervical lymph node signaling after stroke. Nat. Commun. 10, 5306 (2019).

14. Chen, J. et al. Cerebrovascular Injuries Induce Lymphatic Invasion into Brain Parenchyma to Guide Vascular Regeneration in Zebrafish. Dev. Cell 49, 697-710.e5 (2019).

15. Hsu, M., Sandor, M. \& Fabry, Z. Current concepts on communication between the central nervous system and 
peripheral immunity via lymphatics: what roles do lymphatics play in brain and spinal cord disease pathogenesis? Biologia Futura vol. 72 45-60 (2021).

16. Bolte, A. C. et al. Meningeal lymphatic dysfunction exacerbates traumatic brain injury pathogenesis. Nat. Commun. 11, $4524(2020)$.

17. Xiang, M. et al. A Single-Cell Transcriptional Roadmap of the Mouse and Human Lymph Node Lymphatic Vasculature. Front Cardiovasc Med 7, 52 (2020).

18. Takeda, A. et al. Single-Cell Survey of Human Lymphatics Unveils Marked Endothelial Cell Heterogeneity and Mechanisms of Homing for Neutrophils. Immunity 51, 561-572.e5 (2019).

19. Tewalt, E. F. et al. Lymphatic endothelial cells induce tolerance via PD-L1 and lack of costimulation leading to high-level PD-1 expression on CD8 T cells. Blood 120, 4772-4782 (2012).

20. Tewalt, E. F., Cohen, J. N., Rouhani, S. J. \& Engelhard, V. H. Lymphatic endothelial cells - key players in regulation of tolerance and immunity. Front. Immunol. 3, 305 (2012).

21. Tamburini, B. A., Burchill, M. A. \& Kedl, R. M. Antigen capture and archiving by lymphatic endothelial cells following vaccination or viral infection. Nat. Commun. 5, 3989 (2014).

22. Lucas, E. D. et al. Type 1 IFN and PD-L1 Coordinate Lymphatic Endothelial Cell Expansion and Contraction during an Inflammatory Immune Response. J. Immunol. 201, 1735-1747 (2018).

23. Rouhani, S. J. et al. Roles of lymphatic endothelial cells expressing peripheral tissue antigens in CD4 T-cell tolerance induction. Nat. Commun. 6, 6771 (2015).

24. Lucas, E. D. \& Tamburini, B. A. J. Lymph Node Lymphatic Endothelial Cell Expansion and Contraction and the Programming of the Immune Response. Front. Immunol. 10, 36 (2019).

25. Santambrogio, L., Berendam, S. J. \& Engelhard, V. H. The Antigen Processing and Presentation Machinery in Lymphatic Endothelial Cells. Front. Immunol. 10, 1033 (2019).

26. Herz, J., Louveau, A., Da Mesquita, S. \& Kipnis, J. Morphological and Functional Analysis of CNS-Associated Lymphatics. Methods Mol. Biol. 1846, 141-151 (2018).

27. Kretschmer, S. et al. Autofluorescence multiphoton microscopy for visualization of tissue morphology and cellular dynamics in murine and human airways. Lab. Invest. 96, 918-931 (2016).

28. Maruyama, K. et al. Inflammation-induced lymphangiogenesis in the cornea arises from CD11b-positive macrophages. J. Clin. Invest. 115, 2363-2372 (2005). 
29. Zumsteg, A. et al. Myeloid cells contribute to tumor lymphangiogenesis. PLoS One 4, e7067 (2009).

30. Kerjaschki, D. The crucial role of macrophages in lymphangiogenesis. Journal of Clinical Investigation vol. 115 2316-2319 (2005).

31. Trapnell, C. et al. The dynamics and regulators of cell fate decisions are revealed by pseudotemporal ordering of single cells. Nat. Biotechnol. 32, 381-386 (2014).

32. Qiu, X. et al. Single-cell mRNA quantification and differential analysis with Census. Nat. Methods 14, 309-315 (2017).

33. Qiu, X. et al. Reversed graph embedding resolves complex single-cell trajectories. Nat. Methods 14, 979-982 (2017).

34. Volk-Draper, L. D., Hall, K. L., Wilber, A. C. \& Ran, S. Lymphatic endothelial progenitors originate from plastic myeloid cells activated by toll-like receptor-4. PLoS One 12, e0179257 (2017).

35. Ran, S. \& Volk-Draper, L. Lymphatic Endothelial Cell Progenitors in the Tumor Microenvironment. Adv. Exp. Med. Biol. 1234, 87-105 (2020).

36. Tammela, T. \& Alitalo, K. Lymphangiogenesis: Molecular mechanisms and future promise. Cell 140, 460-476 (2010)

37. Alitalo, K. The lymphatic vasculature in disease. Nature Medicine vol. 17 1371-1380 (2011).

38. Giladi, A. et al. Dissecting cellular crosstalk by sequencing physically interacting cells. Nat. Biotechnol. 38, 629-637 (2020).

39. Bendall, S. C. Diamonds in the doublets. Nature biotechnology vol. 38 559-561 (2020).

40. Paddock, C., Zhou, D., Lertkiatmongkol, P., Newman, P. J. \& Zhu, J. Structural basis for PECAM-1 homophilic binding. Blood 127, 1052-1061 (2016).

41. Harris, M. G. et al. Immune privilege of the CNS is not the consequence of limited antigen sampling. Scientific Reports vol. 4 (2015).

42. Rayasam, A. et al. Regional Distribution of CNS Antigens Differentially Determines T-Cell Mediated Neuroinflammation in a CX3CR1-Dependent Manner. The Journal of Neuroscience vol. 38 7058-7071 (2018).

43. Marelli-Berg, F. M., Clement, M., Mauro, C. \& Caligiuri, G. An immunologist's guide to CD31 function in T-cells. Journal of Cell Science vol. 126 2343-2352 (2013).

44. Acton, S. E. et al. Podoplanin-rich stromal networks induce dendritic cell motility via activation of the C-type lectin receptor CLEC-2. Immunity 37, 276-289 (2012). 
45. Johnson, L. A. et al. Dendritic cells enter lymph vessels by hyaluronan-mediated docking to the endothelial receptor LYVE-1. Nat. Immunol. 18, 762-770 (2017).

46. Torzicky, M. et al. Platelet endothelial cell adhesion molecule-1 (PECAM-1/CD31) and CD99 are critical in lymphatic transmigration of human dendritic cells. J. Invest. Dermatol. 132, 1149-1157 (2012).

47. Fletcher, J. M., Lalor, S. J., Sweeney, C. M., Tubridy, N. \& Mills, K. H. G. T cells in multiple sclerosis and experimental autoimmune encephalomyelitis. Clinical \& Experimental Immunology vol. 162 1-11 (2010).

48. Astarita, J. L., Acton, S. E. \& Turley, S. J. Podoplanin: emerging functions in development, the immune system, and cancer. Front. Immunol. 3, 283 (2012).

49. Lane, R. S. et al. IFN $\gamma$-activated dermal lymphatic vessels inhibit cytotoxic T cells in melanoma and inflamed skin. Journal of Experimental Medicine vol. 215 3057-3074 (2018).

50. Sosa, R. A., Murphey, C., Robinson, R. R. \& Forsthuber, T. G. IFN- $\gamma$ ameliorates autoimmune encephalomyelitis by limiting myelin lipid peroxidation. Proc. Natl. Acad. Sci. U. S. A. 112, E5038-47 (2015).

51. Arellano, G., Ottum, P. A., Reyes, L. I., Burgos, P. I. \& Naves, R. Stage-Specific Role of Interferon-Gamma in Experimental Autoimmune Encephalomyelitis and Multiple Sclerosis. Front. Immunol. 6, 492 (2015).

52. Weller, R. O., Sharp, M. M., Christodoulides, M., Carare, R. O. \& Møllgård, K. The meninges as barriers and facilitators for the movement of fluid, cells and pathogens related to the rodent and human CNS. Acta Neuropathol. 135, 363-385 (2018).

53. Norwood, J. N. et al. Anatomical basis and physiological role of cerebrospinal fluid transport through the murine cribriform plate. Elife 8, (2019).

54. Rustenhoven, J. et al. Functional characterization of the dural sinuses as a neuroimmune interface. Cell 184, 1000-1016.e27 (2021).

55. Russo, E., Nitschké, M. \& Halin, C. Dendritic cell interactions with lymphatic endothelium. Lymphat. Res. Biol. 11, $172-182(2013)$.

56. Clarkson, B. D. et al. CCR7 deficient inflammatory Dendritic Cells are retained in the Central Nervous System. Sci. Rep. 7, 42856 (2017).

57. Bianchi, R. et al. Postnatal Deletion of Podoplanin in Lymphatic Endothelium Results in Blood Filling of the Lymphatic System and Impairs Dendritic Cell Migration to Lymph Nodes. Arterioscler. Thromb. Vasc. Biol. 37, $108-117$ (2017). 
58. Johnson, L. A. et al. An inflammation-induced mechanism for leukocyte transmigration across lymphatic vessel endothelium. J. Exp. Med. 203, 2763-2777 (2006).

59. Rinaldi, E. \& Baggi, F. LYVE-1 is 'on stage' now: an emerging player in dendritic cell docking to lymphatic endothelial cells. Cellular \& molecular immunology vol. 15 663-665 (2018).

60. Yao, S. et al. PD-1 on dendritic cells impedes innate immunity against bacterial infection. Blood 113, 5811-5818 (2009).

61. Keir, M. E., Butte, M. J., Freeman, G. J. \& Sharpe, A. H. PD-1 and its ligands in tolerance and immunity. Annu. Rev. Immunol. 26, 677-704 (2008).

62. Park, S. J. et al. Negative role of inducible PD-1 on survival of activated dendritic cells. J. Leukoc. Biol. 95, 621-629 (2014).

63. Krempski, J. et al. Tumor-infiltrating programmed death receptor-1+ dendritic cells mediate immune suppression in ovarian cancer. J. Immunol. 186, 6905-6913 (2011).

64. Lim, T. S. et al. PD-1 expression on dendritic cells suppresses CD8 T cell function and antitumor immunity. Oncoimmunology 5, e1085146 (2016).

65. Palle, P., Monaghan, K. L., Milne, S. M. \& Wan, E. C. K. Cytokine Signaling in Multiple Sclerosis and Its Therapeutic Applications. Med Sci (Basel) 5, (2017).

66. Clarkson, B. D. et al. CCR2-dependent dendritic cell accumulation in the central nervous system during early effector experimental autoimmune encephalomyelitis is essential for effector T cell restimulation in situ and disease progression. J. Immunol. 194, 531-541 (2015).

67. Hauglund, N. L., Kusk, P., Kornum, B. R. \& Nedergaard, M. Meningeal Lymphangiogenesis and Enhanced Glymphatic Activity in Mice with Chronically Implanted EEG Electrodes. J. Neurosci. 40, 2371-2380 (2020).

68. Yu, G., Wang, L.-G., Han, Y. \& He, Q.-Y. clusterProfiler: an R Package for Comparing Biological Themes Among Gene Clusters. OMICS: A Journal of Integrative Biology vol. 16 284-287 (2012). 
A

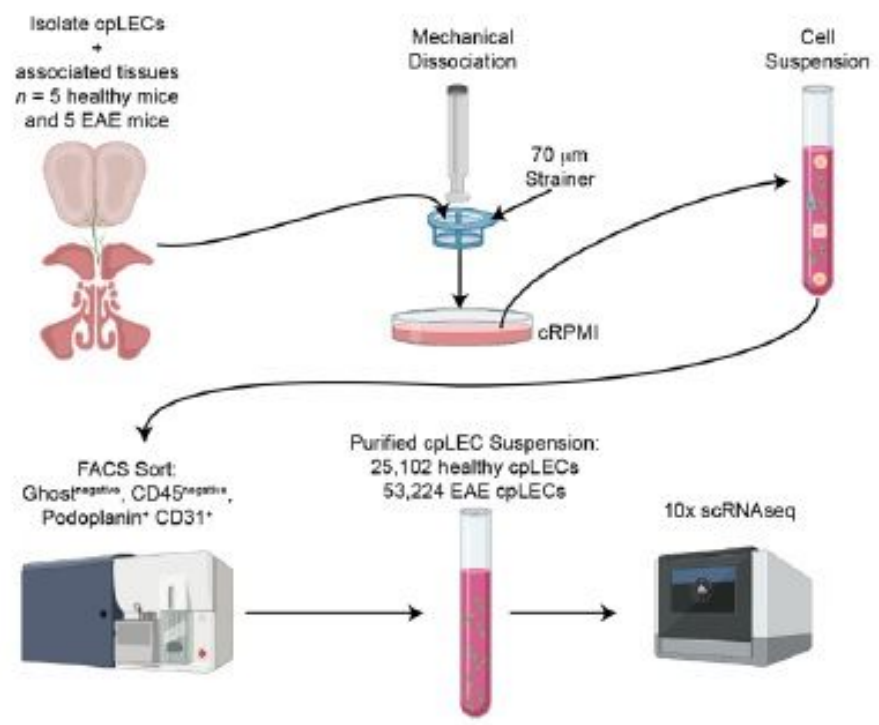

D

Cluster 1

(= $63.01 \%$ of total cells $)$

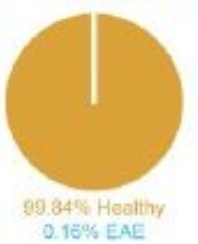

E

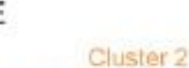

$i=35.35 \%$ of total cells $)$

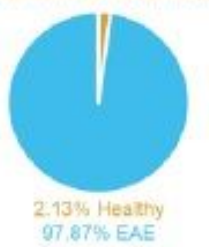

F

Cluster 3

( $=1.64 \%$ of total cells)

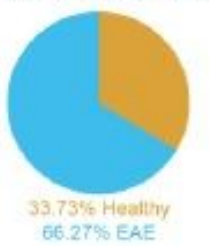

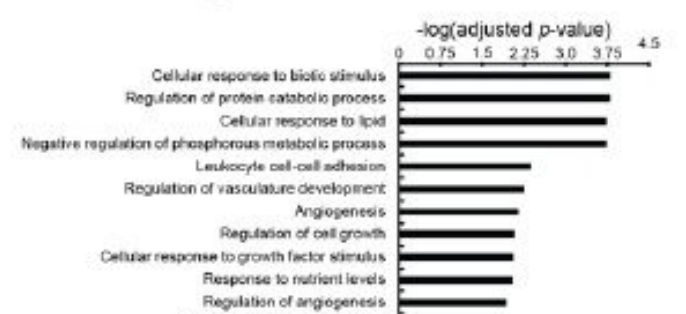

Reguiance of cell colt athesion

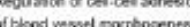

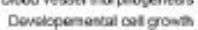
Regunton of creada n nytron
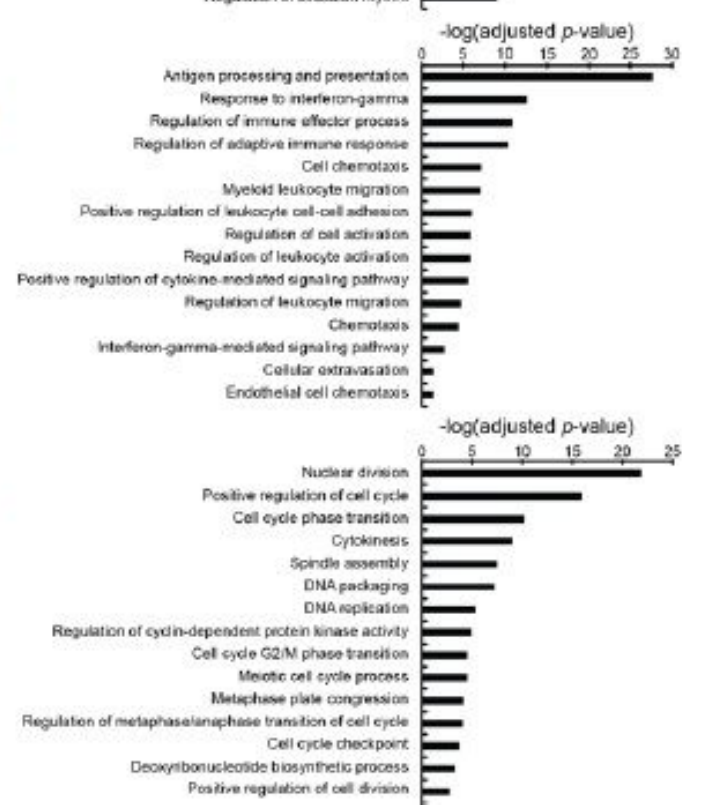

B Healthy vs. EAE
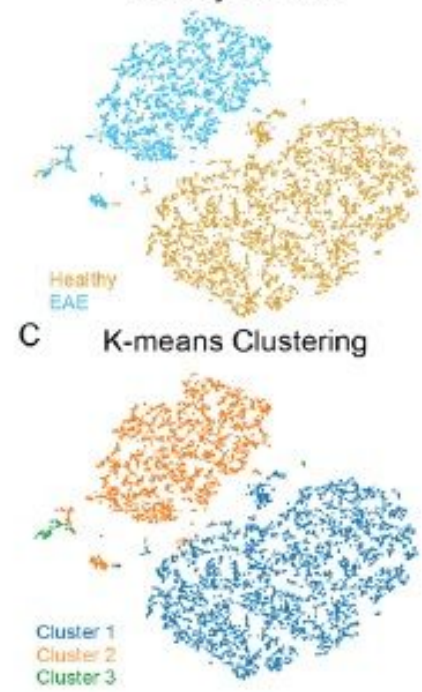

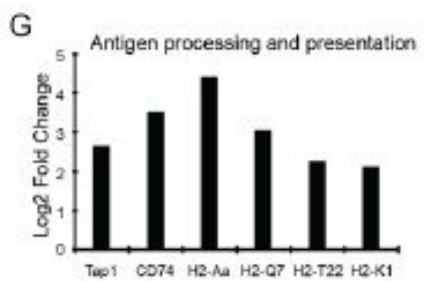

$\mathrm{H}$
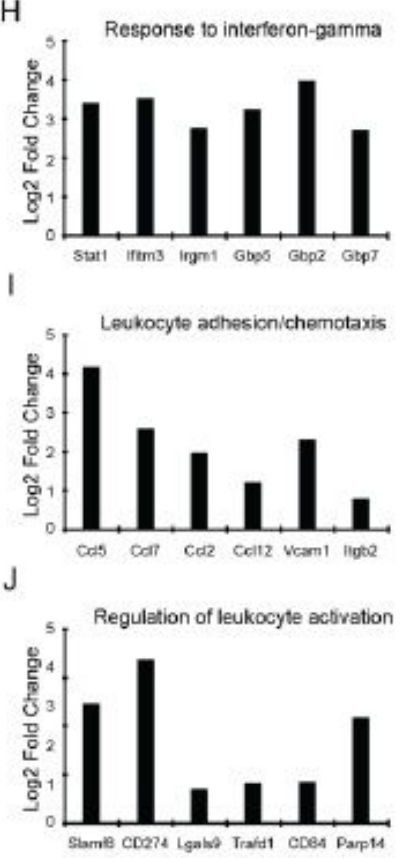

Figure 1

cpLECs display a unique phenotypic signature during neuroinflammation. (A-J): Cells from the cribriform plate were pooled together from 5 healthy mice or 5 EAE mice, and cpLECs were enriched after FACS sorting as previously described for scRNA-seq. (A): Schematic outlining the processing of cpLECs into a 
single cell suspension for scRNA-seq. (B): t-SNE plot of the scRNA-seq dataset explored using Cell Loupe Browser. Healthy and EAE cells are color coded and are separated spatially after excluding non-LECs. (C): Cells were clustered using traditional K-means clustering into 3 unique populations. (D): Left panel: pie chart showing the sources of cells that make up Cluster 1 , which consists primarily of healthy cells. Middle panel: bar graph depicts representative gene ontology pathways that are significantly enriched in this cluster. (E): Left panel: pie chart showing the sources of cells that make up Cluster 2, which consists primarily of EAE cells. Middle panel: bar graph depicts representative gene ontology pathways that are significantly enriched in this cluster. (F): Left panel: pie chart showing the sources of cells that make up Cluster 2, which consists of twice as many EAE cells as healthy. Middle panel: bar graph depicts representative gene ontology pathways that are significantly enriched in this cluster. (G-J): Gene ontology pathways that are significantly upregulated in Cluster 2 were subdivided into four main categories: antigen processing and presentation $(G)$, response to interferon gamma $(H)$, leukocyte adhesion/chemotaxis (I), and regulation of leukocyte activation $(\mathrm{J})$. Bar graphs show 6 representative differentially enriched genes for each category. 


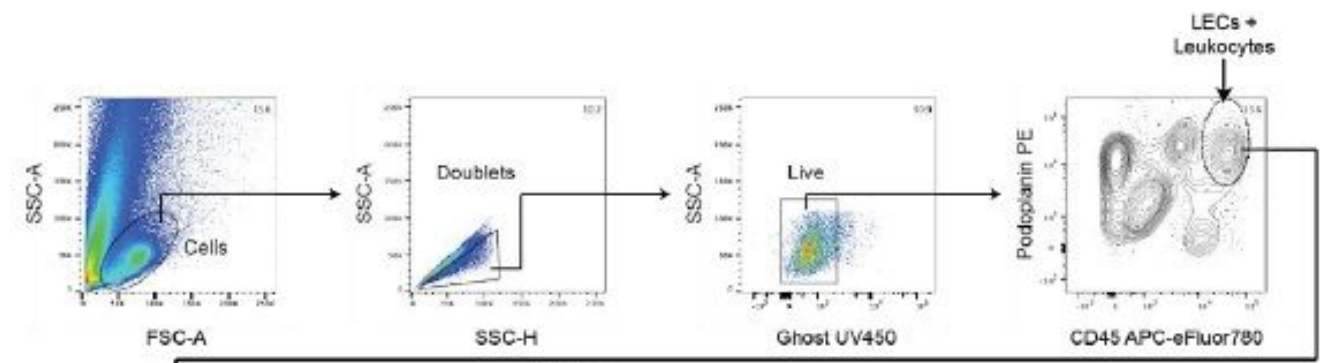

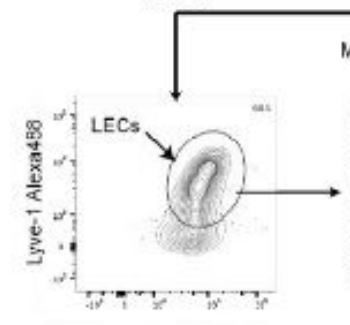

CD31 Alexa647

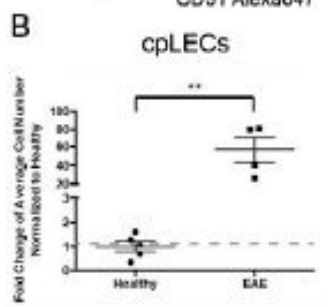

CD8 ${ }^{+} \mathrm{T}$ Cells

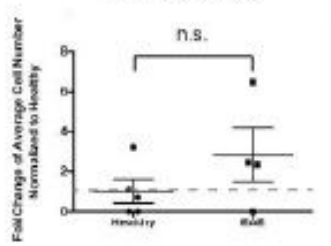

D

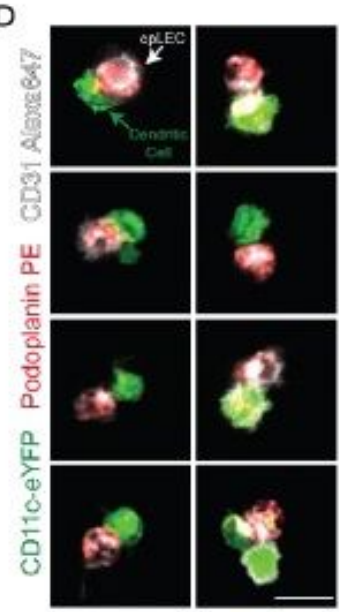

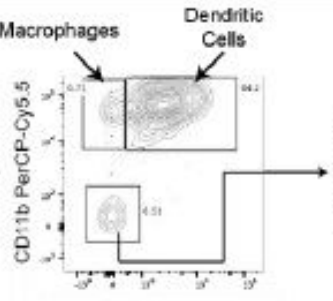

CD 110 BV421

Dendritic Cells

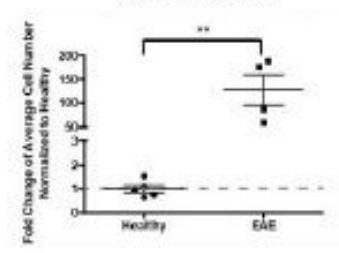

B Cells

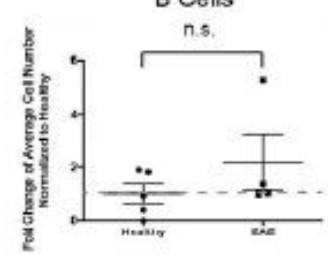

C

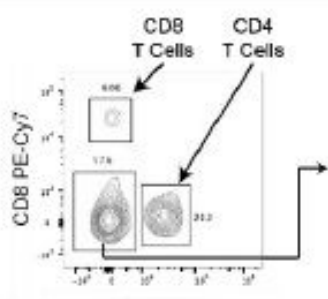

CD4 BUV496

Macrophages

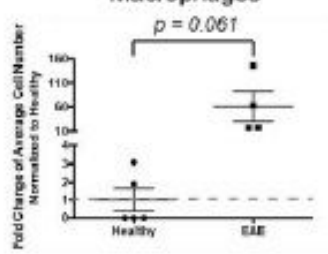

$E$
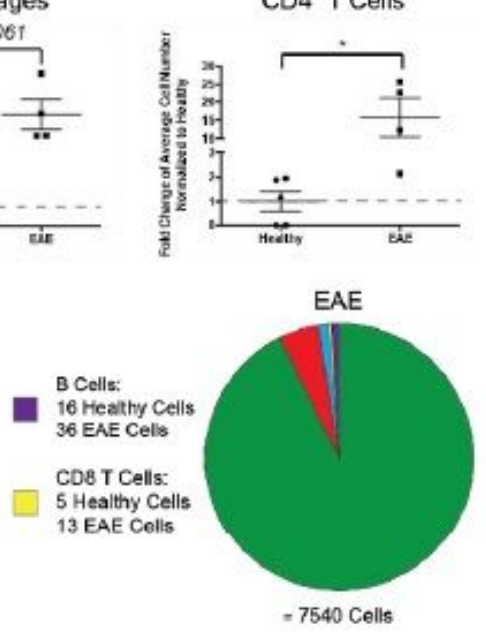

B220 BV521

$\mathrm{CD4}^{+} \mathrm{T}$ Cells
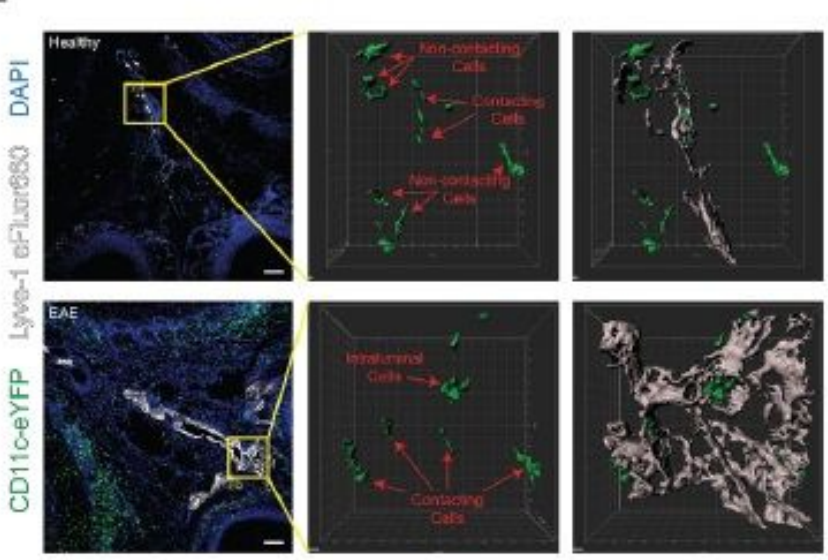

\section{Figure 2}

Leukocytes bind and interact with cpLECs during EAE. (A): Gating strategy used to visualize cpLECleukocyte binding. Doublets consisting of two or more cells bound to each other were gated for live Ghost UV450- cells, and cpLECs bound to leukocytes were gated as CD45+, Podoplanin+, CD31+, and Lyve-1+. Leukocytes bound to cpLECs were further gated for CD11b+ macrophages, CD11 chigh CD11b+ dendritic cells, CD4+ T cells, CD8+ T cells, and B220+ B cells. (B): Quantitation of the average fold changes of 
cpLECs bound to dendritic cells, macrophages, CD4 T cells, CD8 T cells, and B cell numbers during EAE relative to healthy. $n=4-5$ mice per group; data are represented as mean $+/$ - s.e.m., ${ }^{\star} p<0.05,{ }^{*} p<0.01$, unpaired Student's t-test. (C): Pie chart showing the relative composition of the different leukocytes bound to cPLECs during EAE. The legend also states average cell numbers during both healthy and EAE. (D): CD11c-eYFP transgenic reporter mice were induced with EAE and underwent the same harvest protocol as (A-C) to visualize leukocyte-LEC binding using confocal microscopy. Representative confocal microscopy images show doublets and the occasional triplet consisting primarily of a CD11chigh dendritic cell bound to a CD31+ Podoplanin + cpLEC. Scale bar $=20 \mu \mathrm{m}$. (E): CD11c-eYFP transgenic reporter mice were induced with $E A E$, and dendritic cell-cpLEC interactions were visualized in situ using immunohistochemistry. Confocal images of a representative healthy control (top panel) and EAE (bottom panel) underwent 3D surface volume rendering using IMARIS to visualize non-contacting, contacting, and/or intraluminal dendritic cells. Scale bar $=100 \mu \mathrm{m}$. 

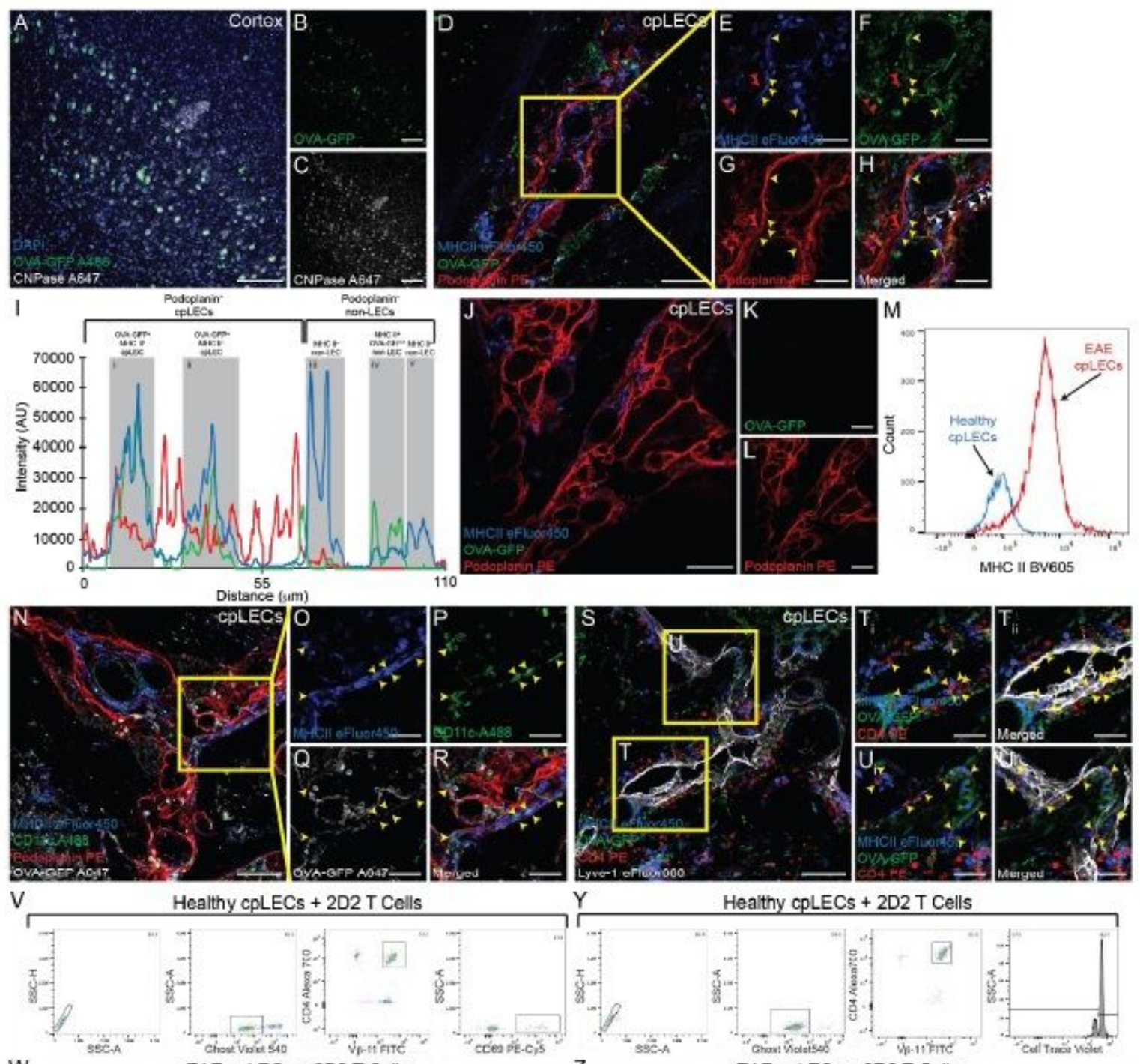

2D2 T Cells
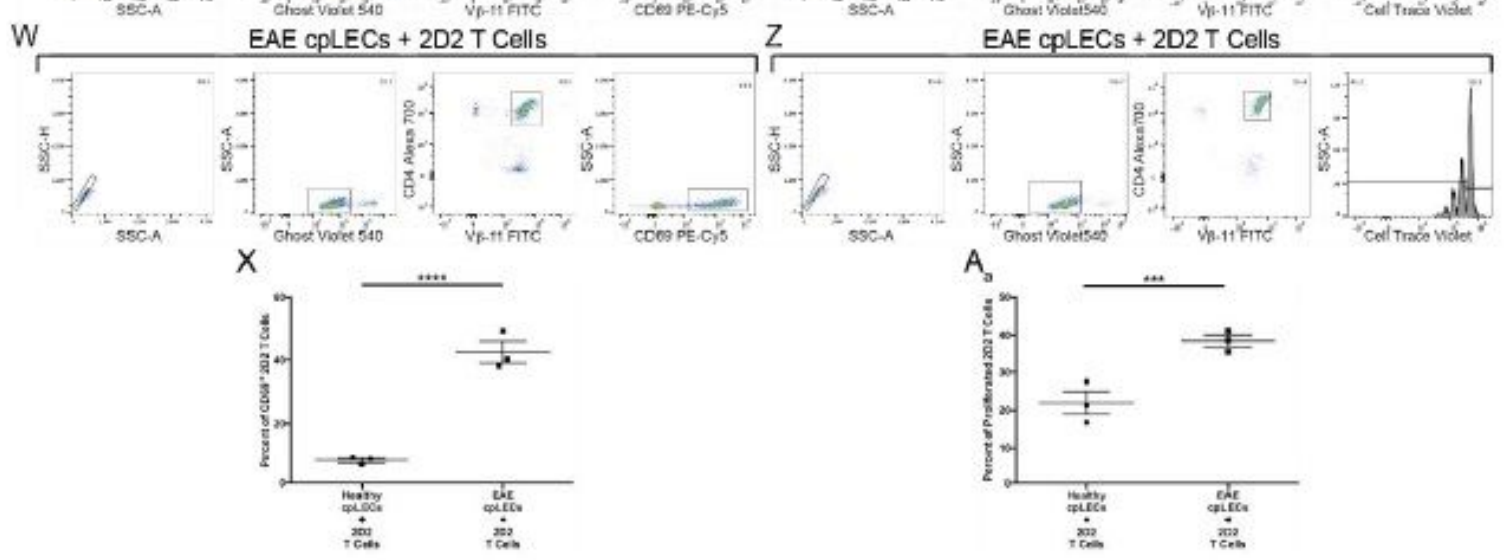

$A_{s}$

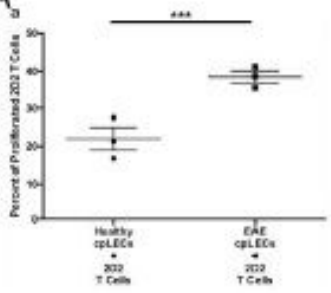

Figure 3

cpLECs have access to CNS-derived antigens and can functionally activate CD4 T cells (A-C): EAE was induced in CNP-OVA transgenic mice, in which OVA-GFPfl/fl expression is driven by the oligodendrocyte specific CNPase-Cre. Immunohistochemistry of layers V/VI of the cortex confirms OVA-GFP expression by CNPase+ oligodendrocytes within the CNS when immunolabeled with an anti-GFP booster antibody. Scale bars $=100 \mu \mathrm{m}(A), 50 \mu \mathrm{m}(\mathrm{B}, \mathrm{C}) .(\mathrm{D}-\mathrm{H})$ : Representative confocal image of Podoplanin $+\mathrm{cpLEC}$ 
uptake of OVA-GFP+ signal and MHC II expression during EAE. Red arrowheads indicate MHC II- OVAGFP+ cpLECs, and yellow arrowheads indicate MHC II+ OVA-GFP+cpLECs. Scale bars $=100 \mu \mathrm{m}(\mathrm{D}), 50$ $\mu \mathrm{m}(\mathrm{E}-\mathrm{H})$. (I): Representative plot profile intensity of cells as indicated by the white line shown in panel $(H)$, which consists of 5 representative cells containing MHC II+ OVA-GFP+ cpLECs (i and ii), MHC II+ noncpLECs (iii and v), and MHC II+ OVA-GFP+ non-cpLECs (iv). (J-L): Control immunolabeling of Podoplanin+ cpLECs with MHC II and without a GFP booster primary antibody, confirming that there is no nonspecific labeling in the GFP channel with the secondary antibody. Scale bars $=100 \mu \mathrm{m}$. (M): Representative histogram of MHC II expression by cpLECs from a healthy or EAE animal after gating for singlets, Ghostnegative live cells, CD31+, and Podoplanin+ cpLECs as previously described. (N-R): Representative confocal image of CD11chigh dendritic cells expressing MHC II and containing OVA-GFP+ signal near and within Podoplanin+ cpLECs. Yellow arrowheads indicate MHC II+ OVA-GFP+ CD11chigh dendritic cells in contact with Podoplanin+ cpLECs. Scale bars $=100 \mu \mathrm{m}(\mathrm{N}), 50 \mu \mathrm{m}(\mathrm{O}-\mathrm{R})$. (S-Uii): Representative confocal image of CD4+ T cells near and within MHC II+ Podoplanin+ cpLECs containing intracellular OVA-GFP signal. Yellow arrowheads indicate CD4+ T cells in contact with MHC II+ OVA-GFP+ Podoplanin + cpLECs. Scale bars $=100 \mu \mathrm{m}(\mathrm{S}), 50 \mu \mathrm{m}$ (Ti-Uii). (V-X): FACS-purified cpLECs from either healthy $(\mathrm{V})$ or EAE (W) mice were co-cultured with purified 2D2 T cells to measure the antigen presentation capability of cpLECs by measuring the early T cell activation marker CD69 after 24 hours. EAE-primed cpLECs are able to activate 2D2 $\mathrm{T}$ cells to a greater extent than healthy cpLECs as measured by percent of 2D2 T cell expression of CD69 (X). Data is represented as mean +/- standard error of the mean, ${ }^{\star \star \star \star} p<0.0001$, unpaired Student's t-test. (Y-Aa): FACS-purified cpLECs from either healthy $(Y)$ or EAE (Z) mice were co-cultured with purified 2D2 T cells labeled with Cell Trace Violet to measure the antigen presentation capability of cpLECs by measuring the proliferation of 2D2 $T$ cells through the dilution of Cell Trace Violet after 72 hours. EAE-primed cpLECs are able to activate 2D2 T cells to a greater extent than healthy cpLECs as measured by increased 2D2 T cell proliferation (Aa). Data is represented as mean $+/$ - standard error of the mean, ${ }^{\star \star \star} \mathrm{p}<0.001$, unpaired Student's t-test. 


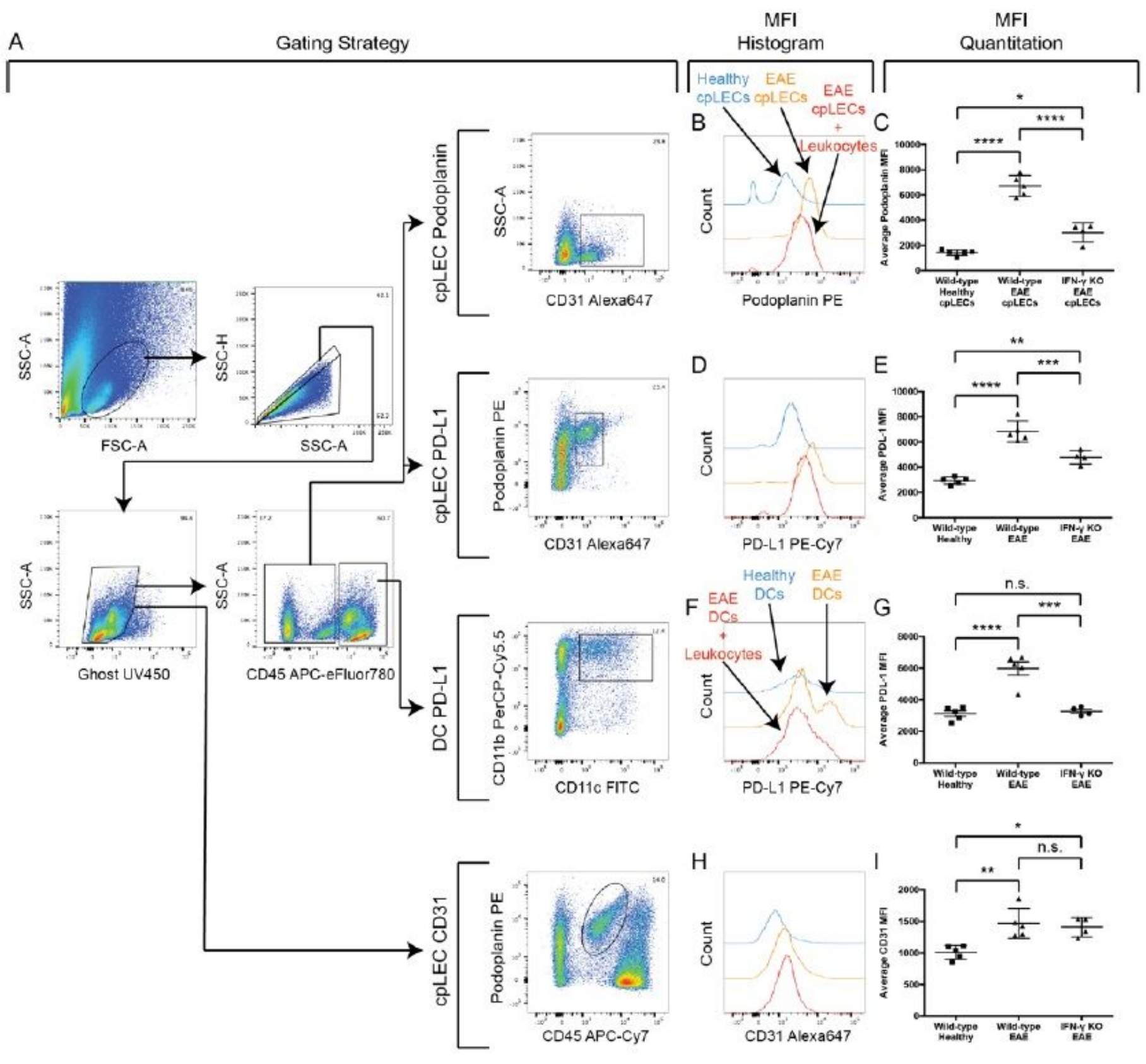

\section{Figure 4}

IFN- $\gamma$ regulates the expression of Podoplanin and PD-L1 during EAE (A): Representative gating strategy used to characterize IFN- $\gamma$ dependent regulation of Podoplanin and PD-L1. cpLECs from healthy wild-type mice, wild-type mice induced with EAE, and IFN- - -/- mice induced with EAE were analyzed for the expression of Podoplanin, PD-L1, and CD31 by flow cytometry. (B-I): The median fluorescence intensity (MFI) of Podoplanin by cpLECs (B-C), PD-L1 by cpLECs (D-E), PD-L1 by dendritic cells (F-G), and CD31 by cpLECs $(\mathrm{H}-\mathrm{I})$ were analyzed. $\mathrm{n}=4-5$ mice per group, data are represented as mean $+/$ - standard error of the mean, ${ }^{*} p<0.05,{ }^{\star \star} p<0.01,{ }^{* \star} \mathrm{p}<0.001,{ }^{\star \star \star *} \mathrm{p}<0.0001$, one-way ANOVA with Tukey's post-hoc multiple comparisons test. 

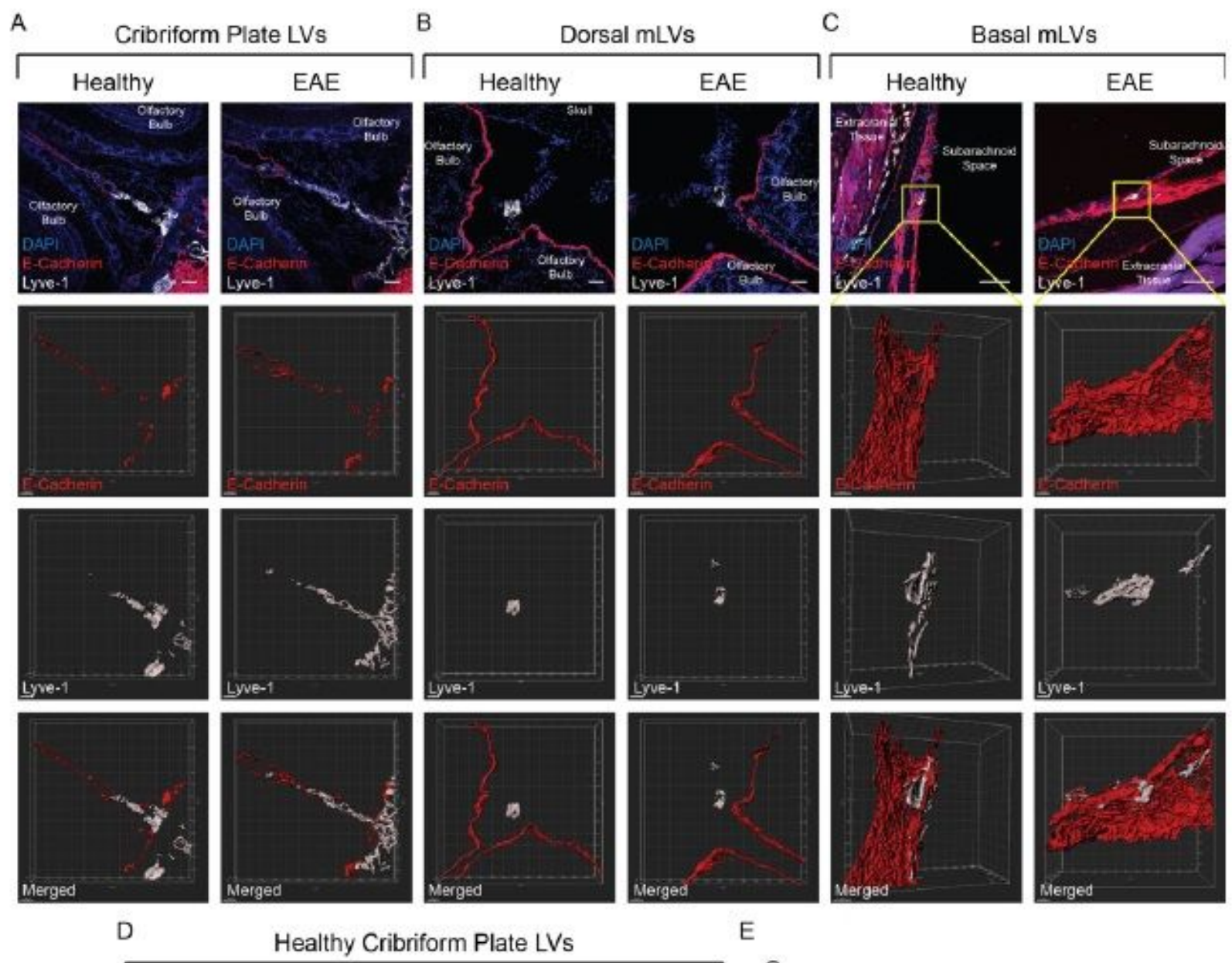

E
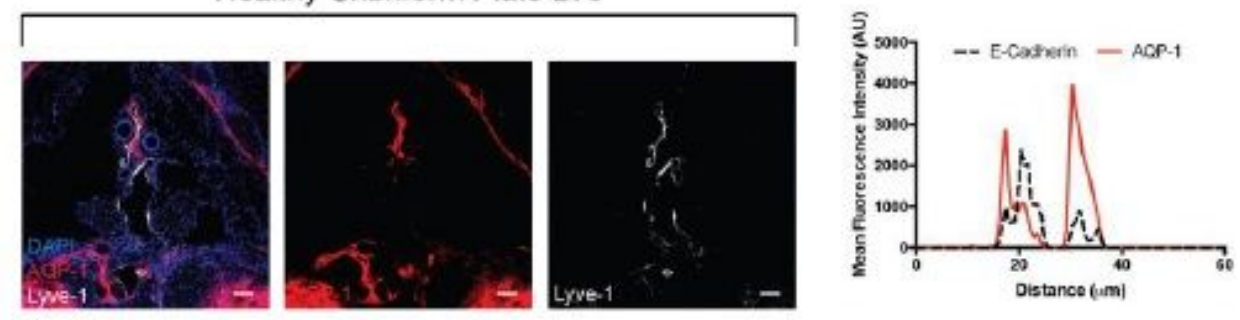

$\mathrm{F}$

EAE Cribriform Plate LVs

G
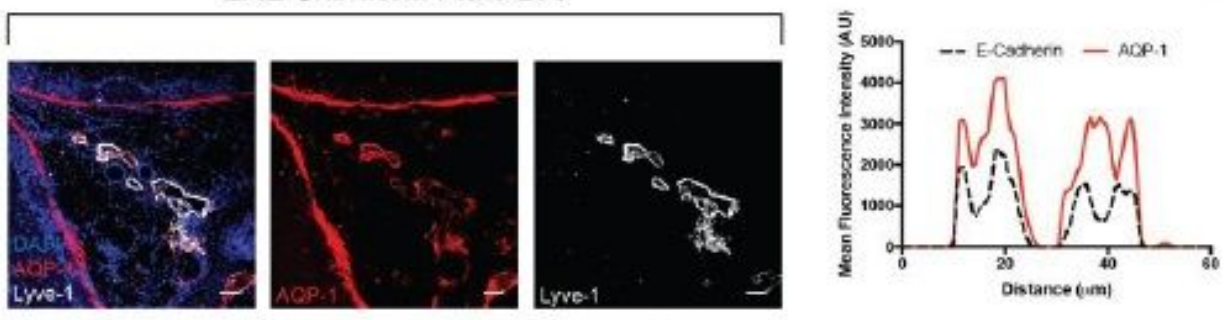

Figure 5

cpLECs have access to the subarachnoid space and express AQP-1 (A-C): Immunolabeling of the cribriform plate (A), dura above the olfactory bulbs (B), and basal meninges below the brain (C) from coronal sections of the whole head. Top row: merged immunohistochemistry images labeled with DAPI to visualize nuclei, E-Cadherin to visualize the epithelial cells that comprise the arachnoid barrier, and Lyve-1 for lymphatic vessels. Second row: 3D surface volume rendering using IMARIS of the E-Cadherin+ 
arachnoid barrier. Third row: 3D surface volume rendering of Lyve-1+lymphatic vessels. Fourth row: 3D surface volume rendering of both the E-Cadherin+ arachnoid barrier with Lyve-1+ lymphatic vessels. Scale bars $=100 \mu \mathrm{m}$. (D-G): Immunolabeling of cpLECs from either healthy (D) or EAE (F) with Lyve-1 and the water channel AQP-1. Representative plot profile intensity analysis confirms co-localization of AQP-1 expression by Lyve-1+ cpLECs in both healthy (E) and EAE (G). Scale bars $=100 \mu \mathrm{m}$.

A
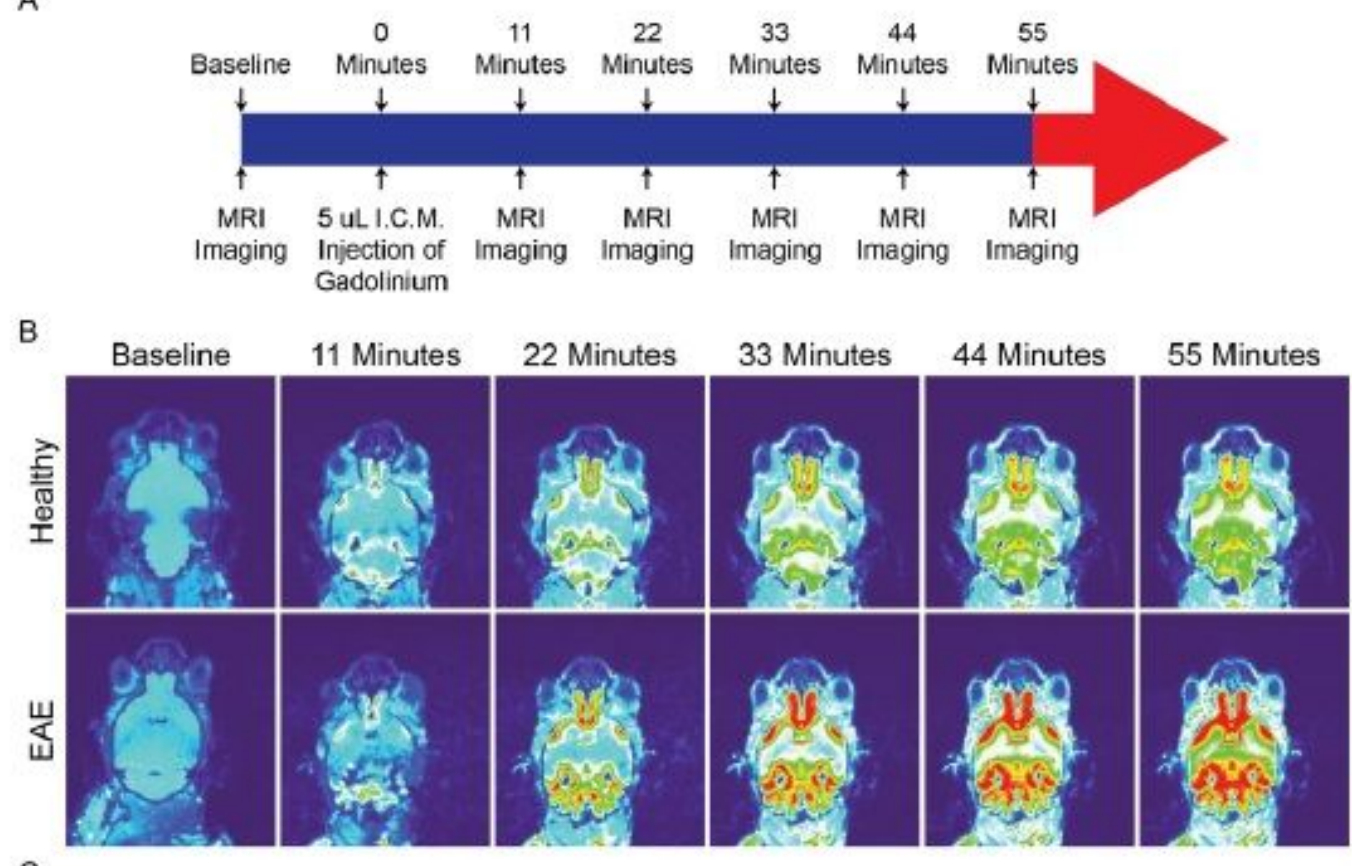

C
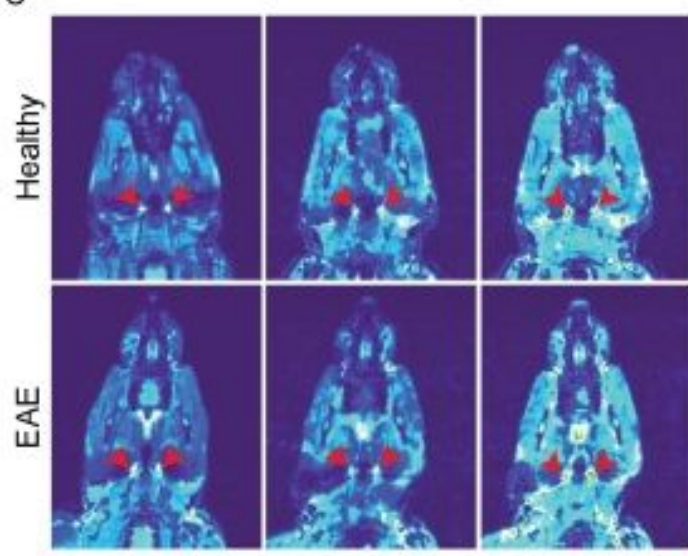

D

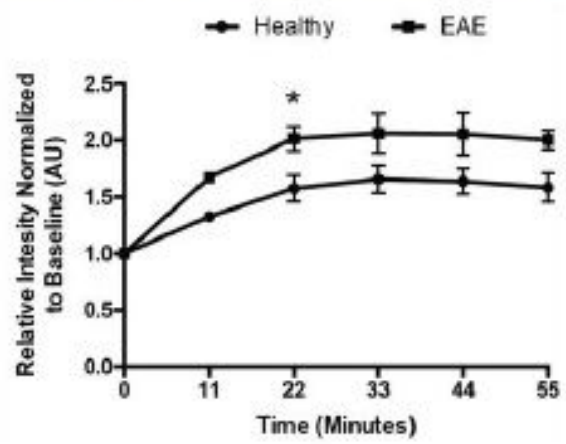

$\mathrm{E}$
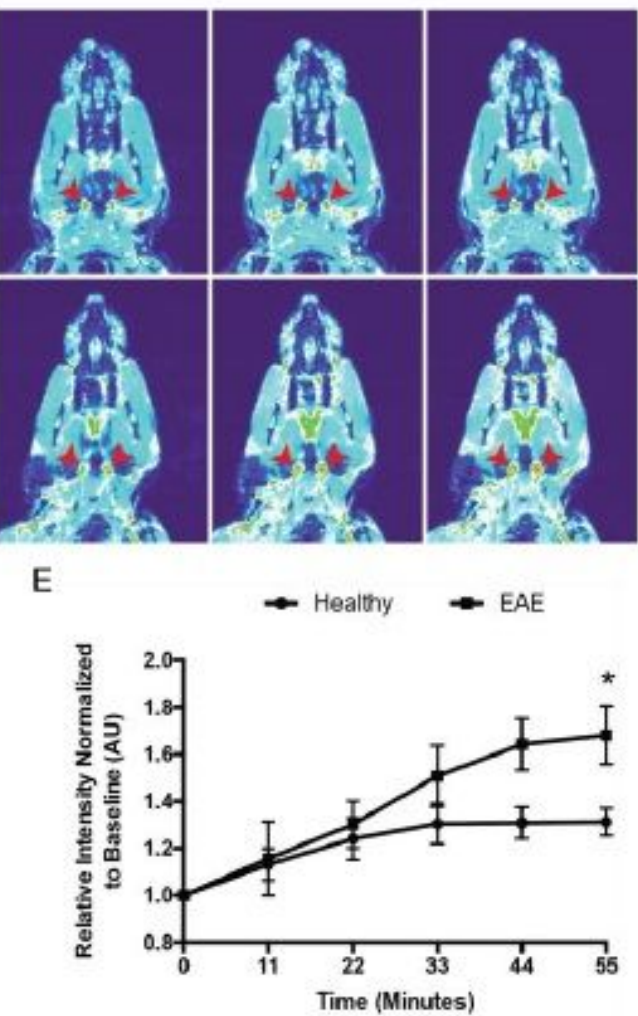

Figure 6 
CPLECs have access to a CSF reservoir (A): Schematic of experimental design for visualizing CSF dynamics using MRI in healthy and EAE mice. (B): Representative T1-weighted scans of the base of the brain and olfactory bulbs where the basal meningeal lymphatics and cPLECs reside at baseline before Gadolinium infusion into the cisterna magna, and serial T1-weighted scans over time after Gadolinium infusion. Note the accumulation of Gadolinium signal between the olfactory bulbs and base of the brain. (C): Representative scans of the nec showing the dCLNs as indicated by the red arrowheads. (D): Quantitation of the average pixel intensity where cpLECs reside, ventral medially to the olfactory bulbs normalized to baseline between healthy and EAE. $n=4$ mice per group; data are represented as mean +/standard error of the mean, ${ }^{*} p<0.05$, repeated two-way ANOVA using Sidak's multiple comparisons test. (E): Quantitation of the average pixel intensity of the dCLNs as indicated by the arrowheads in (C) normalized to baseline between healthy and EAE. $n=4$ mice per group, data are represented as mean $+/$ standard error of the mean, ${ }^{*} p<0.05$, repeated two-way ANOVA using Sidak's multiple comparisons test.

\section{Supplementary Files}

This is a list of supplementary files associated with this preprint. Click to download.

- SupplementaryFigures.pdf 